\title{
Probabilistic optimisation of mono-stringer composite stiffened panels in post-buckling regime
}

\author{
Hamed Farokhi $^{1} \cdot$ Omar Bacarreza $^{2} \cdot$ M.H. Ferri Aliabadi ${ }^{2}$ \\ Received: 31 March 2019 / Revised: 9 February 2020 / Accepted: 3 March 2020 / Published online: 15 May 2020 \\ (C) The Author(s) 2020
}

\begin{abstract}
In this paper, a multi-objective probabilistic design optimisation approach is presented for reliability and robustness analysis of composite structures and demonstrated on a mono-omega-stringer stiffened panel. The proposed approach utilises a global surrogate model of the composite structure while accounting for uncertainties in material properties as well as geometry. Unlike the multi-level optimisation approach which freezes some parameters at each level, the proposed approach allows for all parameters to change at the same time and hence ensures global optimum solutions in the given parameter design space (for both probabilistic and deterministic optimisations) within a certain degree of accuracy. The proposed approach is used in this study to conduct extensive multi-objective probabilistic and deterministic optimisations (without considering safety factors) on a mono-stringer stiffened panel. In particular, a global surrogate model is developed utilising the computational power of a highperformance computing facility. The inputs of the surrogate model are the omega-stringer geometry and the mechanical properties of the composite material, while the outputs are the fundamental linear buckling load (LBL) and the nonlinear postbuckling strength (NPS). LBL and NPS are obtained via detailed parametric finite element models of the mono-stringer stiffened panel; in the nonlinear model, the interface between the skin and the omega-stringer is modelled via cohesive elements to allow for debonding in the post-buckled regime. Extensive multi-objective optimisations are conducted on the surrogate model using deterministic and probabilistic approaches to examine the omega-stringer geometric parameters mostly affecting the system robustness and reliability. The differences between deterministic and probabilistic designs are highlighted as well.
\end{abstract}

Keywords Probabilistic design $\cdot$ Multi-objective optimisation $\cdot$ Surrogate modelling $\cdot$ Post-buckling regime $\cdot$ Stiffened panel

\section{Introduction}

Carbon fibre-reinforced composites are widely used in aircraft structures. The recent advancements in composites allowed the industry to increase their usage of composite materials dramatically. To put it into numbers, the latest models by Boeing and Airbus are made of 50 and $53 \%$ composites, respectively. These percentages will grow in the future to even further reduce the mass of the aircraft while achieving higher

Responsible Editor: W. H. Zhang

Hamed Farokhi

hamed.farokhi@northumbria.ac.uk

1 Department of Mechanical and Construction Engineering, Northumbria University, Newcastle upon Tyne NE1 8ST, UK

2 Department of Aeronautics, Imperial College London, London SW7 2AZ, UK strengths and increased lifespan. One of the key elements of an aircraft is the composite stiffened panel, in which the skin is reinforced via adding a stringer for superior load carrying in both tension and compression. The main concern in the design of a stiffened panel, for sections of an aircraft under compression, is maximising the compressive load carrying due to the buckling phenomenon (Hao et al. 2017). For the case of omega-stringer stiffened panels, the initial buckling starts in the skin (Kassapoglou 2013; Wang and Abdalla 2015), here referred to as the fundamental linear buckling load (LBL); however, it is known that beyond the first linear buckling, a stiffened panel could carry loads of several times the magnitude of the fundamental LBL before failure, here referred to as the nonlinear post-buckling strength (NPS). Hence, designing a panel which will always operate below the fundamental LBL is very conservative; given the extra strength of the panel in the post-buckling regime, much lighter designs can be achieved by allowing the panel to operate in the postbuckling regime. This has motivated a large amount of 
research on stiffened panel optimisation in the post-buckling regime. In what follows, a concise review of the vast literature on optimisation of composite structures is given; more detailed reviews can be found in surveys conducted by, for instance, Venkataraman and Haftka (1999), Ghiasi et al. (2009) Ghiasi et al. (2010) and Nikbakt et al. (2018).

The traditional approach of optimisation, also known as deterministic optimisation (DO), does not account for any uncertainties in the system. Modern optimisation techniques, also known as probabilistic optimisation (PO), on the other hand, account for uncertainties (Salas and Venkataraman 2008) that could affect the design objectives, such as uncertainties associated with mechanical properties or the geometry. Two well-stablished PO techniques are robust-design optimisation (RDO) and reliability-based design optimisation (RBDO) (Chen and Qiu 2018; das Neves Carneiro and Antonio 2018; Fang et al. 2018; Hu and Duan 2018; Kaveh et al. 2018; López et al. 2017; Montoya et al. 2015; Sohouli et al. 2018; Strömberg 2017). RDO focuses on minimising the sensitivity of the objective function to random changes in the uncertain variables in the system, while RBDO aims at achieving a certain confidence in reliability of the product under a prescribed probabilistic constraint. In what follows, a brief literature review is conducted on different optimisation techniques.

One of the methods of optimising stiffened panels is the finite strip method (FSM) originally developed by Wittrick (1968) and Cheung (1968), in which the stiffened panel is divided into a finite number of strips and the motions of the strips are approximated via trigonometric functions. Further research was conducted using FSM technique for instance by Bushnell (1987), Butler and Williams (1993) and Zabinsky (1998). Homogenisation-based methods have also been used for optimisation of stiffened panels (Wang and Abdalla 2016; Wang et al. 2017; 2018; 2019). For instance, Wang et al. (2018) conducted a sensitivity analysis for optimisation of non-uniform curved stiffened composite panels in the framework of homogenisation-based local/global analysis.

The finite element (FE) technique is another method for modelling and analysis of stiffened panels which is superior to FSM. However, accurate FE models, which account for geometric nonlinearities, progressive failure and interfacial debonding, are usually very time consuming. Conducting a multi-objective optimisation (Coello 2000; Marler and Arora 2004; Zitzler et al. 2000) of stiffened panel using FE models could become very time consuming as massive computational power is required to search the multi-dimensional design space while accounting for different sources of nonlinearities. To address this problem, a multi-level approach has been suggested by Sobieszczanski-Sobieski et al. (1987) in which the large optimisation problem is divided into levels of substructures. A two-level optimisation procedure for a composite wing was developed by Liu et al. (2000), who constructed response surface for the optimal buckling load at the lower level and employed it later for global optimisation. Herencia et al. (2008a) proposed a two-level approach for layup optimisation of composite stiffened panels. A multi-level approach was developed by Wind et al. (2008), who conducted local and global optimisation on a multi-component structure. Further studies were conducted by Herencia et al. (2008b) who proposed a two-step method for optimisation of anisotropic composite stiffened panels. Bacarreza et al. (2015) employed a multilevel approach to conduct robust-design optimisation on composite stiffened panels in post-buckling regime. Although multi-level approaches reduce the computational costs, they do not guarantee a global optimum solution as in each level there is only a selection of system parameters which act as optimisation inputs while the rest are kept fixed.

Another technique for reducing the computational costs is to use a surrogate model (Albanesi et al. 2018; Marhadi and Venkataraman 2008) to approximate the response of the system. Lamberti et al. (2003) investigated the use of approximate models for conducting global optimisation on stiffened panels and concluded that it allows a greater exploration of the global design space compared to the case of utilising local optimisation together with complicated models. Having developed a surrogate model, different algorithms can be utilised to perform deterministic or probabilistic optimisation. Venkataraman and Salas (2007) proposed an approach for studying the mechanics influencing progressive failure predictability and developing surrogate models for deterministic optimisation in order to maximise performance. Barkanov et al. (2014) conducted linear buckling optimisation analysis on composite lateral wing upper covers utilising the response surface method together with optimal Latin hypercube sampling. In the second part of the study (Barkanov et al. 2016), the optimum designs based on linear buckling analysis were verified through a nonlinear buckling analysis and re-optimised if necessary; they utilised the response surface technique for surrogate modelling and optimisation purposes. Other studies have been performed on stiffened panel deterministic optimisation using surrogate modelling, for instance, by Bisagni and Lanzi (2002), Lanzi and Giavotto (2006), Irisarri et al. (2011) and Marín et al. (2012). López et al. (2017) conducted deterministic and reliability-based design optimisations of composite stiffened panels in post-buckling regime; they conducted a decoupled RBDO which separates the reliability analysis from the deterministic optimisation. Further RBDO studies of stiffened panel have been conducted by, for instance, Qu and Haftka (2003), who conducted RBDO and computed the reliability constraints employing Monte Carlo sampling and a design response surface, and Díaz et al. (2016), who performed a comparison of stochastic expansions and moment-based methods 
for the reliability analysis while using genetic and gradient-based techniques for deterministic optimisation.

The present study first proposes a highly reliable multiobjective probabilistic design optimisation approach through development of a global surrogate model and then applies that approach to a stiffened panel. More specifically, the proposed approach is detailed in Section 2. Then in Sections 3, 4 and 5, the approach is demonstrated for a mono-omega-stringer stiffened panel. The main advantage of the proposed approach over multi-level optimisation approaches is that it allows for all parameters to change simultaneously and hence ensures more general (near-global) probabilistic and deterministic optimum solutions. It is worth noting that in this study, a safety factor is not considered for either probabilistic or deterministic optimisations since the goal here is to differentiate between the underlying mechanism of these optimisation approaches, which comes before application of any safety factors.

\section{Global multi-objective probabilistic design optimisation approach}

The FE method has proven to be the most reliable technique for analysis of aircraft structures. The increased accuracy offered by FE techniques comes at the price of increased computational cost, which depending on the size and complexity of the structure could vary between hours to weeks of run time. Hence, conducting even deterministic optimisation on aircraft structures could be a computationally challenging task with months of run time. Now in the context of probabilistic optimisation which requires a large number of objective function calls (in the order of millions), it becomes impossible to use the original FE model as the objective function. In such cases, it is inevitable to use a surrogate model as an explicit approximation of the original FE model. Surrogate models have been used extensively for optimisation purposes in different fields. In the case of aircraft structures, surrogate models are usually used with very limited number of inputs and outputs in multi-level approaches to reduce computational costs; however, since some parameters are kept fixed at each level, the multi-level approaches do not guarantee global optimum result. In this study, a new surrogate model-based probabilistic optimisation approach is presented which is more efficient and more accurate than the multi-level approaches since it allows for all parameters to change simultaneously. The steps of this approach are explained for a general case in the following.

\subsection{Developing a parametric FE model}

The first step is developing a parametric FE model with a specific number of inputs and outputs. Although in general there is no specific limit on the number of inputs and outputs of the surrogate model of a structure, the proposed approach focuses on the main contributing input parameters of the structure under consideration to reduce the computational costs and to increase accuracy. The mechanical properties of the composite structure $\left(E_{11}, E_{22}=E_{33}, G_{23}\right.$ and $\left.G_{12}=G_{13}\right)$ should always be considered as inputs since there are always uncertainties associated with their value and they could significantly affect the desired output. Apart from these four inputs, the geometric parameters which mostly affect the desired output should be considered as well. The number of geometric inputs could vary depending on the structure under consideration. After determining the desired inputs, detailed parametric FE models should be developed which calculate the outputs for a given set of inputs.

\subsection{Creating matrix of design samples}

Design-of-experiments methods are commonly utilised to determine the spatial distribution of samples in the design space. The main goal of design-of-experiments is to maximise the amount of information obtained from a limited number of sample points (Koziel et al. 2011). More specifically, design-of-experiments will provide a matrix of design samples which best represents the whole design space. In the proposed approach, the preferred method to create the matrix of design samples is the optimal Latin hypercube sampling technique (Park 1994). There is no general formula for the number of samples based on the number of inputs and outputs, as it depends on the sensitivity of the outputs to variations of the inputs and many other factors. The proposed approach suggests a sample size of at least 10 times the number of inputs; however, increasing the number of samples will reduce the error later on when constructing the surrogate model. After creating a matrix of design samples, the parametric FE models of the structure should be used to calculate the desired outputs for each sample in the design space. This step is the most computationally expensive part of the simulation. Parallelising simulation of the samples could decrease the total run time significantly.

\subsection{Surrogate model development}

Different software packages and codes are available for surrogate model development. In the present approach, the Surrogate Modelling (SUMO) toolbox (Gorissen et al. 2010) is suggested for this purpose due to its comprehensive library of algorithms. The SUMO toolbox is capable of generating surrogate models based on various algorithms and functions such as kriging, artificial neural network (ANN), radial basis functions, extreme machine learning and genetic algorithm, just to name a few. Hence, it provides a platform to generate and test various surrogate models for the system under 
consideration. Additionally, it offers different measures for error analysis such as cross-validation and reference data comparison. Having developed the matrix of design samples including inputs and outputs within a specific range of parameters, various surrogate models offered by the SUMO toolbox can be tested to find the one which offers the most accuracy. The accuracy of a surrogate model can be examined via two different measures, i.e. cross-validation and direct comparison to a unique data set (Gorissen et al. 2010; Rikards et al. 2006). In a situation where the desired accuracy is not met, further design samples should be created and computed using the FE models to increase the size of the matrix of the design samples. This should be repeated until the desired accuracy is met.

\subsection{Global multi-objective optimisation}

Having developed the surrogate model of the system, the final step is to conduct multi-objective probabilistic and deterministic optimisations on the surrogate model. Genetic algorithms are best suited for multi-objective optimisations. The main advantage of the proposed approach is that it offers a direct comparison between probabilistic and deterministic optimisation results. Deterministic optimisation, robust-design optimisation and reliability-based design optimisation are conducted on the developed surrogate model.

A general robust-design optimisation problem can be mathematically expressed as:

$\min \{\mu(F(\mathbf{d}, \mathbf{x})), \sigma(F(\mathbf{d}, \mathbf{x}))\}$

subject to : $g_{m}(\mathbf{d}) \leq 0, m=1,2, \ldots, M$,

in which $F$ stands for the objective function, $\mathbf{d}$ and $\mathbf{x}$ represent the design and random variables, $\mu$ and $\sigma$ show the mean value and the standard deviation and $g_{m}$ denotes the $m$ th constraint. It should be noted that in an RDO, randomness could be associated with either design variables or other system variables. For an aircraft structure, uncertainties could be associated with mechanical properties, i.e. $E_{11}, E_{22}=E_{33}, G_{23}$ and $G_{12}=G_{13}$, and/or geometric parameters. Again, the global surrogate model of the structure allows for accounting for uncertainties in any of the inputs.

In a reliability-based design optimisation, on the other hand, the uncertainties are usually associated with mechanical properties. The RBDO process ensures that the final design meets a certain probabilistic constraint up to a prescribed reliability level $\beta_{t}$. In general, an RBDO problem can be formulated as

$\min F(\mathbf{d})$

subject to :

$$
\begin{aligned}
& g_{m}(\mathbf{d}) \leq 0, m=1,2, \ldots, M, \\
& P\left[G_{n}(\mathbf{d}, \mathbf{x}) \leq 0\right]-P_{\mathrm{f}, n} \leq 0, n=1,2, \ldots, N,
\end{aligned}
$$

where $\mathbf{d}$ and $\mathbf{x}$ are the design and random variables, respectively, and $F$ is the objective function. $g_{m}$ denotes $m$ th deterministic constraint, while $G_{n}$ stands for the $n$th probabilistic one. $P[]$ represents the probability of the constraint being met, with $P_{\mathrm{f}}$ being the allowable probability of failure. For the case when the random variables are characterised by a normal distribution, $P_{\mathrm{f}}$ is related to the prescribed reliability level $\beta_{t}$ via $P_{\mathrm{f}}=\Phi\left(-\beta_{t}\right)$ in which $\Phi$ is the cumulative distribution function of the standard normal distribution (Enevoldsen and Sørensen 1994).

Different steps of the general approach detailed in this section are shown in Fig. 1. In what follows, the approach proposed in this section is demonstrated in detail on a monostringer stiffened panel.

\section{Demonstration of the proposed optimisation approach on a mono-stringer stiffened panel}

Consider a mono-stringer stiffened panel of length $L$ and width $W$, as shown in Fig. 2 a. The omega-stringer geometry is detailed in Fig. 2 b, with $X_{1}, X_{2}, H$ and $\theta$ being the foot, flange, height and angle, respectively. The mono-stringer is clamped at both ends, with the righthand end being free to move in the longitudinal direction (shown by $z$ in sub-figure a). Furthermore, there are no constraints on the two longitudinal edges of the skin. In this section, the approach proposed in Section 2 is implemented for this structure. In particular, the surrogate model of the structure will be developed in this section. The optimisation results will be discussed in separate sections.

\subsection{Parametric FE model development}

The inputs of interest are the omega-stringer geometry, i.e. $X_{1}, X_{2}, H$ and $\theta$, as well as the mechanical properties, i.e. $E_{11}, E_{22}=E_{33}, G_{23}$ and $G_{12}=G_{13}$. The desired outputs are linear buckling load as well as the nonlinear post-buckling strength. In this section, two parametric FE models of the mono-stringer stiffened panel are developed in Abaqus as detailed below.

\subsubsection{Linear FE model}

The first parametric FE model developed in this section is a linear eigen-buckling analysis model in order to obtain the fundamental linear buckling load. An axial load is applied to the movable end of the mono-stringer and an eigen-buckling analysis is conducted to obtain the fundamental linear buckling load of the stiffened panel. Conducting a mesh convergence analysis for the linear buckling load shows that a global mesh size of $2 \mathrm{~mm}$ yields reliable results. The model takes 8 


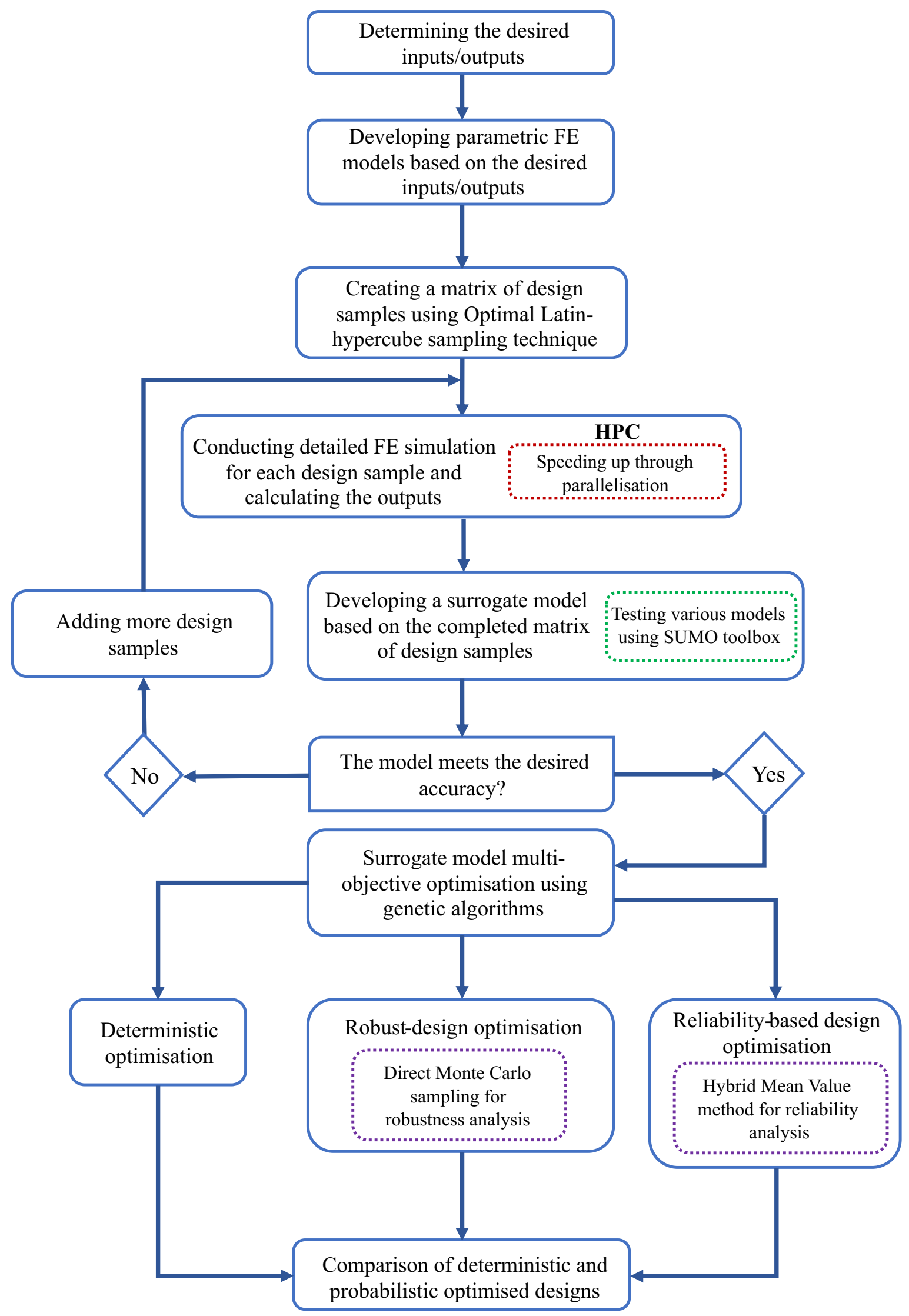

Fig. 1 Flowchart of the proposed approach 
(a)

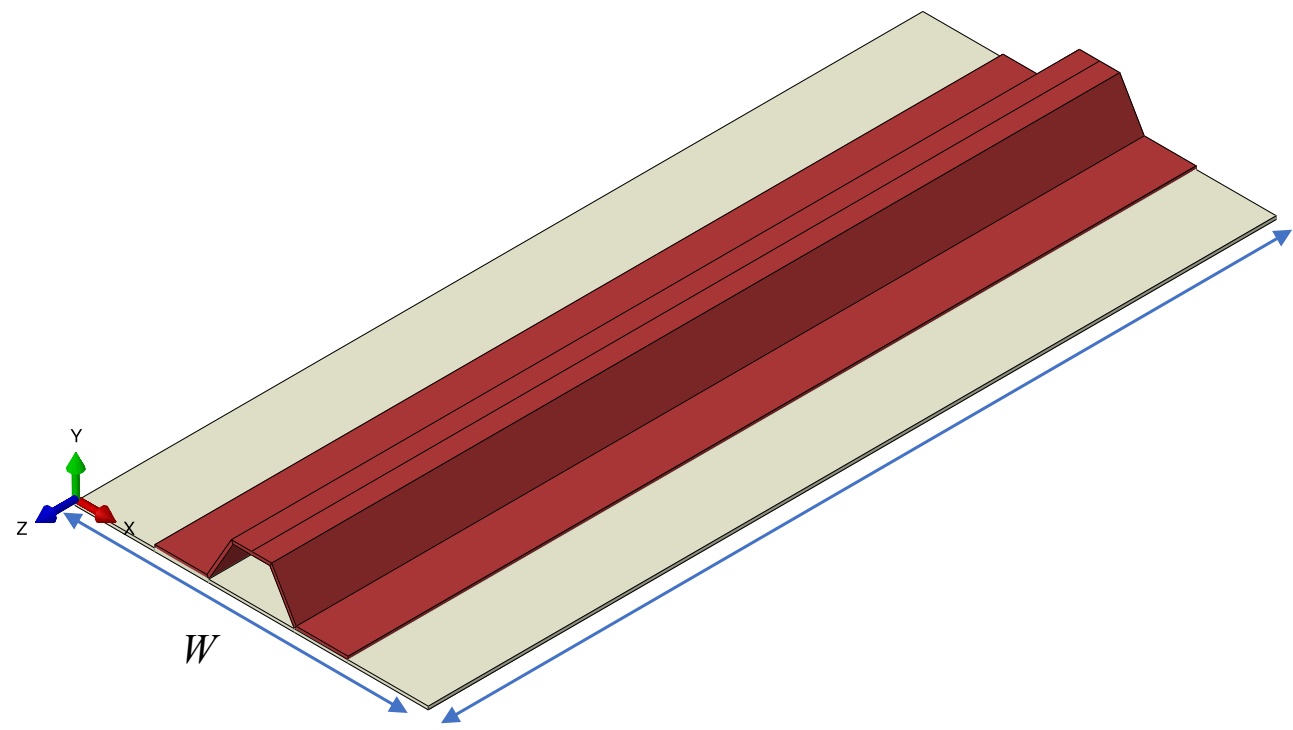

(b)

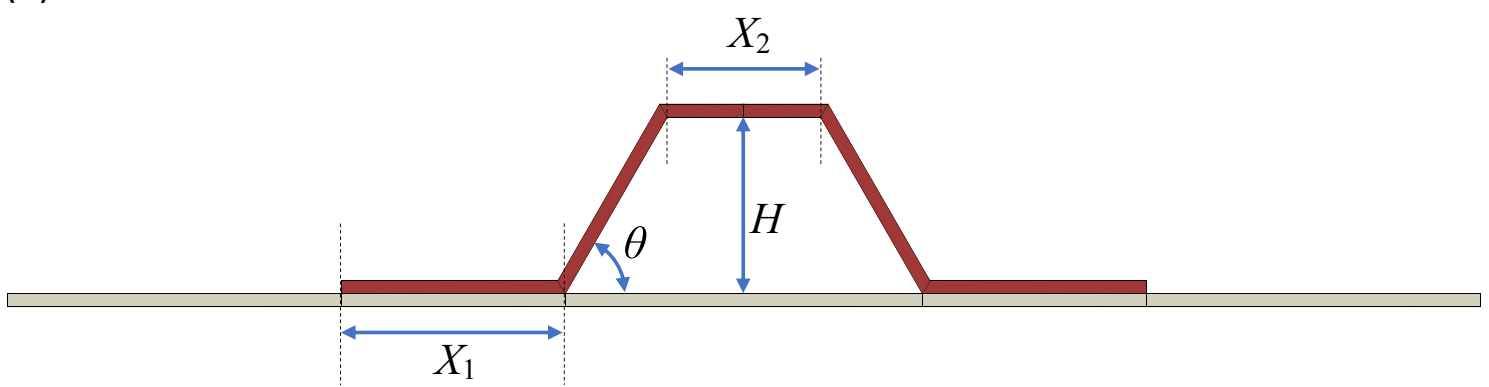

Fig. 2 a Schematic representation of the mono-stringer stiffened panel. b The geometric parameters of the stringer

inputs as described before and calculates the fundamental linear buckling load.

\subsubsection{Nonlinear FE model}

A nonlinear parametric FE model is developed in this section in order to obtain the nonlinear post-buckling strength of the composite stiffened panel. This model is much more complex compared to the first model and accounts for geometric nonlinearities and progressive failure and additionally allows for interfacial debonding via use of cohesive elements. It is known that in the post-buckling regime, the buckled shape of the skin changes continuously due to increased compressive load. Additionally, sudden changes in the modes of buckling, known as mode-switch, occurs in the post-buckling regime which cannot be properly captured via use of quasi-static FE techniques. To this end, the nonlinear explicit dynamic FE analysis is employed in this study for analysing the nonlinear post-buckling characteristics.
Modelling the cohesive elements and correct selection of properties for modelling a traction-separation response is a challenging task. The main reason is that for typical epoxy resin matrix-based carbon fibre-reinforced composites, the length of the cohesive zone is less than $1 \mathrm{~mm}$. An accurate representation of the traction in the tip of the crack and propagation of delamination requires at least three elements in the cohesive zone. Such small mesh requirement demands significant computational costs for structural analysis, such as the case of the mono-stringer stiffened panel of the present study. To address this challenge, the procedure introduced by Turon et al. (2007) is employed in this study, in which the cohesive zone length is artificially increased via decreasing the interfacial strengths; this procedure is briefly explained in the following.

In order to model the cohesive elements based on a mixedmode fracture bilinear traction-separation, the following properties are required: the critical fracture energies $G_{\mathrm{IC}}, G_{\mathrm{IIC}}$ and $G_{\text {IIIC }}$; the penalty stiffnesses $K_{1}, K_{2}$ and $K_{3}$; and the interfacial strengths $\tau^{0}, \mu_{1}^{0}$ and $\mu_{2}^{0}$. In this study, it is assumed that penalty stiffnesses are the same $\left(K_{1}=K_{2}=K_{3}=K\right)$; furthermore, $\mu_{1}^{0}$ 
$=\mu_{2}^{0}=\mu^{0}$ and $G_{\text {IIC }}=G_{\text {IIIC }}$. The penalty stiffness value should be large enough so that it has a negligible influence on the effective elastic properties of the composite. To this end, the penalty stiffness is defined as

$K=\alpha \frac{E_{3}}{t}$,

where $E_{3}$ is the material's transverse Young's modulus, $t$ is the thickness of the adjacent sublaminate and $\alpha$ is a coefficient much larger than 1. Turon et al. (2007) suggest a value more than 50; in the present study, $\alpha=100$ is used.

The cohesive zone lengths for modes I and II can be approximated as (Turon et al. 2007)

$l_{\mathrm{cz}}^{(\mathrm{I})}=M E_{2} \frac{G_{\mathrm{IC}}}{\left(\tau^{0}\right)^{2}}$,

$l_{\mathrm{cz}}^{(\mathrm{II})}=M E_{2} \frac{G_{\mathrm{IIC}}}{\left(\mu^{0}\right)^{2}}$,

where $M$ is a parameter characterised by the employed cohesive zone model; in the present study, Hillerborg's model (Hillerborg et al. 1976) is utilised in which $M=1$.

Denoting the length of the cohesive element by $l_{\mathrm{ce}}$ and assuming $N_{\mathrm{ce}}$ as the number of elements in the cohesive zone, the cohesive zone lengths in (4) can be replaced by $N_{\mathrm{ce}} l_{\mathrm{ce}}$. Under the assumption of linear elastic fracture mechanics, the effect of the interfacial strengths can be neglected. Hence, based on (4), the length of the cohesive zone can be artificially increased via decreasing the interfacial strengths. As a result, the modified interfacial strengths $\left(\tau_{m}\right.$ and $\left.\mu_{m}\right)$ can be obtained as

$\tau_{m}=\sqrt{\frac{M E_{2} G_{\mathrm{IC}}}{N_{\mathrm{ce}} l_{\mathrm{ce}}}}, \mu_{m}=\sqrt{\frac{M E_{2} G_{\mathrm{IIC}}}{N_{\mathrm{ce}} l_{\mathrm{ce}}}}$.

The final values for interracial strengths that ensure $N_{\text {ce }}$ number of elements of size $l_{\text {ce }}$ span the cohesive zone are

$\tau_{f}=\operatorname{Min}\left\{\tau_{m}, \tau^{0}\right\}, \mu_{f}=\operatorname{Min}\left\{\mu_{m}, \mu^{0}\right\}$.

In the present study, $N_{\mathrm{ce}}=3$ and $l_{\mathrm{ce}}=1.0 \mathrm{~mm}$ are selected. Furthermore, a global mesh size of $2.0 \mathrm{~mm}$ is used for both the skin and the stringer. Such selection of mesh size ensures converged and reliable predictions and practical simulation run time.

The properties of the composite and interface materials used in the present study are given in Table 1. The damage initiation is defined based on Hashin criteria (Hashin 1980; Hashin and Rotem 1973); additionally, the damage propagation is governed by the amount of energy dissipated through progressive damage (Lapczyk and Hurtado 2007).

The results of the nonlinear FE simulation for a monostringer of $X_{1}=35.74 \mathrm{~mm}, X_{2}=30.0 \mathrm{~mm}, H=25.0 \mathrm{~mm}$ and $\theta=55.0^{\circ}$ are shown in Fig. 3. In particular, the load- displacement curve of the mono-stringer under compressive load is shown in sub-figure a. As seen, the skin buckling (i.e. linear buckling) occurs in the vicinity of $0.5 \mathrm{~mm}$ shortening, while the failure occurs at around $2.2 \mathrm{~mm}$ shortening (i.e. point A). The mode of failure is skin-stringer debonding; the out-of-plane displacement (i.e. buckling amplitude) of the skin and stringer at points $\mathrm{A}, \mathrm{B}$ and $\mathrm{C}$ are shown in sub-figures $\mathrm{b}$ $\mathrm{d}$, respectively. In this study, the nonlinear post-buckling strength, denoted as NPS, refers to the maximum load the mono-stringer stiffened panel carries before failure, i.e. the load corresponding to point $\mathrm{A}$.

The nonlinear parametric FE model developed in this subsection takes 8 inputs as described previously and calculates the nonlinear post-buckling strength.

\subsection{Creating matrix of design samples}

In this section, the optimal Latin hypercube sampling method is employed to create the matrix of design samples. In particular, 260 sample points are created for the 8 inputs considered with the range of the inputs given in Table 2. As mentioned in Section 2, the size of the cohesive element is set to $1 \mathrm{~mm}$ in this study. To ensure that this size remains fixed for all computer experiments, the foot size of the omega-stringer in the sampling matrix is rounded to the nearest integer. Furthermore, an additional 15-sample test matrix is generated as well via the optimal Latin hypercube sampling for the sole purpose of error analysis.

While the simulations run quite fast for the linear model, the nonlinear model simulation run time (utilising $20 \mathrm{CPU}$ cores) varies between 16 and $20 \mathrm{~h}$ per each sample, depending on the total number of cohesive elements. This means an average total run time of $4680 \mathrm{~h}$ (195 days!) to run all 260 samples. To be able to finish the design of experiments in a reasonable amount of time, the computational power of a high-performance computing (HPC) facility is utilised. In particular, each sample is run on one node consisting of 20 CPUcores and $128 \mathrm{~GB}$ memory, and multiple samples are run at the same time. A $10 \times$ speed up is achieved using the HPC facility and the simulations are finished within almost 20 days. Having developed a $260 \times 10$ design matrix, as well as a $15 \times$ 10 reference design matrix for error analysis, different surrogate models are generated and tested, as explained in the following, to obtain a reliable explicit approximation of the nonlinear and linear FE models. It is important to note that the mode of failure in all the cases examined in this section is skin-stringer debonding which initiates irreversible damage in the structure. In other words, if the skin-stringer debonding is not considered, the stiffened panel could withstand much larger loads; therefore, the skin-stringer debonding is the failure bottleneck for the case of mono-omega-stringer stiffened panel examined in this study. As a result, the nonlinear post- 
Table 1 Material properties

\begin{tabular}{|c|c|c|c|}
\hline & Symbol & Value & Description \\
\hline \multirow{6}{*}{$\begin{array}{l}\text { Elastic properties of the } \\
\text { composite material }\end{array}$} & $E_{11}(\mathrm{GPa})$ & 139.0 & Longitudinal modulus of elasticity \\
\hline & $E_{22}=E_{33}(\mathrm{GPa})$ & 8.1 & Transversal modulus of elasticity \\
\hline & $\nu_{23}$ & 0.45 & In-plane Poisson's ratio \\
\hline & $\nu_{12}=\nu_{13}$ & 0.33 & Out-of-plane Poisson's ratio \\
\hline & $G_{23}(\mathrm{GPa})$ & 4.8 & In-plane shear modulus \\
\hline & $G_{12}=G_{13}(\mathrm{GPa})$ & 3.1 & Out-of-plane shear modulus \\
\hline \multirow{10}{*}{$\begin{array}{l}\text { Damage initiation parameters } \\
\text { of the composite material }\end{array}$} & $\sigma_{1}^{0 \mathrm{t}}(\mathrm{MPa})$ & 2900 & Longitudinal tensile strength \\
\hline & $\sigma_{1}^{0 \mathrm{c}}(\mathrm{MPa})$ & 1660 & Longitudinal compressive strength \\
\hline & $G_{\mathrm{c} 1}^{\mathrm{t}}(\mathrm{N} / \mathrm{mm})$ & 162 & Tensile fracture toughness in the longitudinal direction \\
\hline & $G_{\mathrm{c} 1}^{\mathrm{c}}(\mathrm{N} / \mathrm{mm})$ & 106 & Compressive fracture toughness in the longitudinal direction \\
\hline & $\sigma_{2}^{0 \mathrm{t}}=\sigma_{3}^{0 \mathrm{t}}(\mathrm{MPa})$ & 58 & Transverse tensile strength \\
\hline & $\sigma_{2}^{0 \mathrm{c}}=\sigma_{3}^{0 \mathrm{c}}(\mathrm{MPa})$ & 25 & Transverse compressive strength \\
\hline & $G_{\mathrm{c} 2}^{\mathrm{t}}=G_{\mathrm{c} 3}^{\mathrm{t}}(\mathrm{N} / \mathrm{mm})$ & 0.303 & Tensile fracture toughness in the transversal direction \\
\hline & $G_{\mathrm{c} 2}^{\mathrm{c}}=G_{\mathrm{c} 3}^{\mathrm{c}}(\mathrm{N} / \mathrm{mm})$ & 1.0 & Compressive fracture toughness in the transversal direction \\
\hline & $\sigma_{12}^{0}=\sigma_{13}^{0}(\mathrm{MPa})$ & 125 & Out-of-plane shear strength \\
\hline & $\sigma_{23}^{0}(\mathrm{MPa})$ & 95 & In-plane shear strength \\
\hline \multirow[t]{4}{*}{ Cohesive material properties } & $\tau^{0}(\mathrm{MPa})$ & 75 & Maximum nominal normal stress \\
\hline & $\mu^{0}(\mathrm{MPa})$ & 95 & Maximum nominal shear stress \\
\hline & $G_{\mathrm{IC}}(\mathrm{N} / \mathrm{mm})$ & 0.303 & Normal fracture energy \\
\hline & $G_{\mathrm{IIC}}(\mathrm{N} / \mathrm{mm})$ & 0.651 & Shear fracture energy \\
\hline
\end{tabular}

buckling strength (NPS) considered in this study is in fact the maximum load-carrying capacity without permanent damage.

\subsection{Surrogate model development}

In this section, a global surrogate model is developed in which the omega-stringer geometry $\left(X_{1}, X_{2}, H\right.$ and $\left.\theta\right)$ and the mechanical properties of the composite structure $\left(E_{11}, E_{22}=E_{33}\right.$, $G_{23}$ and $G_{12}=G_{13}$ ) are considered as inputs. The fundamental linear buckling load and the nonlinear post-buckling strengths are considered as outputs. Hence, a surrogate model with 8 inputs and 2 outputs is developed in this section using the $260 \times 10$ design matrix developed in the previous section. The mono-stringer length and width as well as the skin and omega-stringer thickness and layup are kept fixed in this study so as not to further increase the computational costs. In this study, the SUMO toolbox (Gorissen et al. 2010) is utilised to develop a surrogate model. As mentioned before, the SUMO toolbox includes various algorithms and functions such as kriging, ANN, radial basis functions, extreme machine learning and genetic algorithm. Hence, various algorithms can be tested to find one which best suits the present problem. Two measures for error analysis are considered: cross-validation and the 15-sample reference data set that was developed in Section 3.2. Even though the number of samples is fixed, the SUMO toolbox uses an optimisation algorithm and generates iterations of surrogate models until a best model is found. After testing various model building algorithms, it is found that the artificial neural network and kriging models give the best results for the mono-stringer stiffened panel under consideration. It is found that the surrogate model error can be further decreased by mixing the two models, with the coefficient for each model being determined via another optimisation algorithm. The final version of the mixed ANN-kriging surrogate model predicts the nonlinear post-buckling strength with less than $1.5 \%$ error and the fundamental linear buckling load with less than $0.5 \%$ error for the assumed range of inputs. This surrogate model will be used in the following sections as an explicit approximation of the linear buckling load and nonlinear post-buckling strength of the mono-stringer stiffened panel to conduct extensive multi-objective deterministic and probabilistic optimisations.

\section{Multi-objective deterministic optimisation}

This section conducts a multi-objective deterministic optimisation (MODO) on the surrogate model developed in the previous section; as mentioned before, a safety factor is not considered for either deterministic or probabilistic optimisations. In the present study, the Pareto envelope-based selection algorithm II (PESA-II) is utilised to conduct multi-objective 
(a)

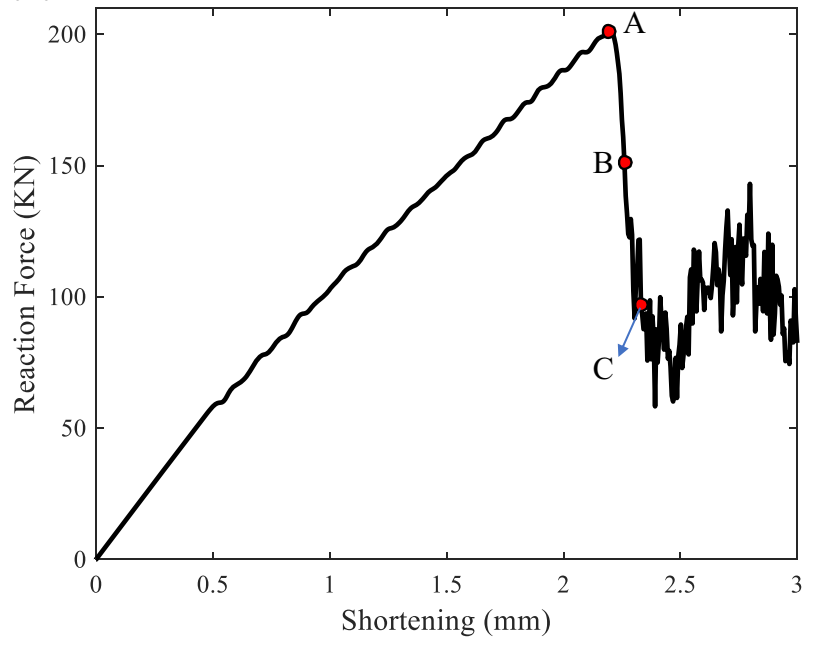

(b)

(c)
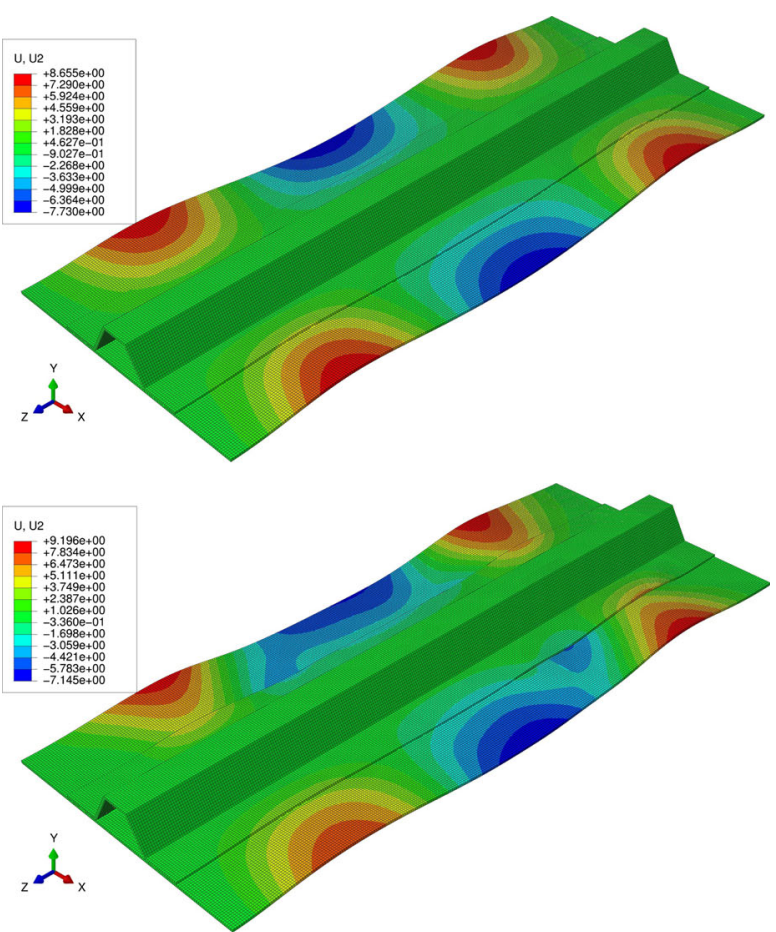

(d)

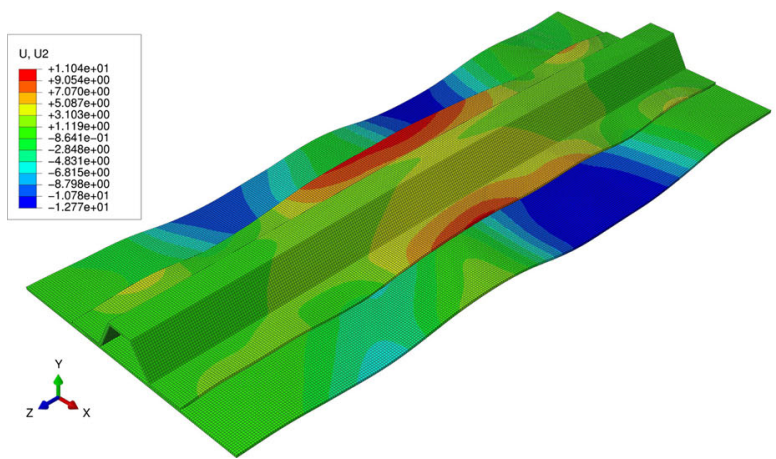

Fig. 3 a Load-displacement curve of the mono-stringer stiffened panel under compressive load. b-d Out-of-plane displacement of the skin and stringer corresponding to points A, B and C of sub-figure a, respectively
Table 2 Fixed and variable parameters

\begin{tabular}{ll}
\hline Fixed parameters & Value \\
Skin and stringer thickness $(\mathrm{mm})$ & 2.208 \\
Skin and stringer layup & {$[45 /-45 / 0 / 0 / 90 / 0]_{\mathrm{s}}$} \\
Panel length, $L(\mathrm{~mm})$ & 600.0 \\
Panel width, $W(\mathrm{~mm})$ & 250.0 \\
Design of experiment inputs & Range \\
Stringer foot, $X_{1}(\mathrm{~mm})$ & $26 \leq X_{1} \leq 46$ \\
Stringer flange, $X_{2}(\mathrm{~mm})$ & $18 \leq X_{2} \leq 30$ \\
Stringer angle, $\theta\left(^{\circ}\right)$ & $55 \leq X_{1} \leq 65$ \\
Stringer height, $H(\mathrm{~mm})$ & $25 \leq H \leq 35$ \\
$E_{11}(\mathrm{GPa})$ & $109 \leq E_{11} \leq 169$ \\
$E_{22}=E_{33}(\mathrm{GPa})$ & $6.4 \leq E_{22} \leq 9.9$ \\
$G_{23}(\mathrm{GPa})$ & $2.4 \leq G_{23} \leq 3.8$ \\
$G_{12}=G_{13}(\mathrm{GPa})$ & $3.8 \leq G_{12} \leq 5.8$ \\
\hline
\end{tabular}

optimisation (Corne et al. 2001; Corne et al. 2000). PESA-II is a multi-objective evolutionary optimisation algorithm which utilises the genetic algorithm approach along with a selection based on the Pareto envelope. Furthermore, it utilises an archive to store the approximate Pareto solutions. Parents and mutants are the chosen from this archive, based on the grids which themselves are generated based on the distribution of the archive members.

The optimisation algorithm aims at minimising the mass of the stringer and maximising both the fundamental linear buckling load and the nonlinear post-buckling strength. The multiobjective optimisation is conducted by giving a specific weight to each of the objectives and then adding/subtracting them such that they reduce into one objective; more details on the weight of the objectives are given in the discussions for each case. Since the aim of this section is to conduct deterministic optimisation, only the geometric parameters of the stringers are treated as variables in the optimisation loop. In other words, the mechanical properties are kept fixed while conducting the optimisation. The goal here is to identify the major differences between the stringer geometries optimised for maximum linear buckling load and those optimised for maximum nonlinear post-buckling strength. It should be noted that since in this study only the geometry of the stringer (and not the skin) is varied in the optimisation loop, the reported mass is that of the stringer only. The skin mass is a constant of $529.9 \mathrm{~g}$ throughout this study.

The results of the 3-objective PESA-II-based optimisation is shown in Fig. 4 through three sub-figures. For the sake of clarity, a 2-dimensional (2D) graph with two vertical axes is used to show the results of the 3-objective optimisation. The horizontal axis shows the mass of the stringer, while the vertical axes illustrate the LBL and NPS objectives. A portion of the solutions obtained by the optimisation algorithm are plotted, with blue dots representing NPS and orange dots showing 

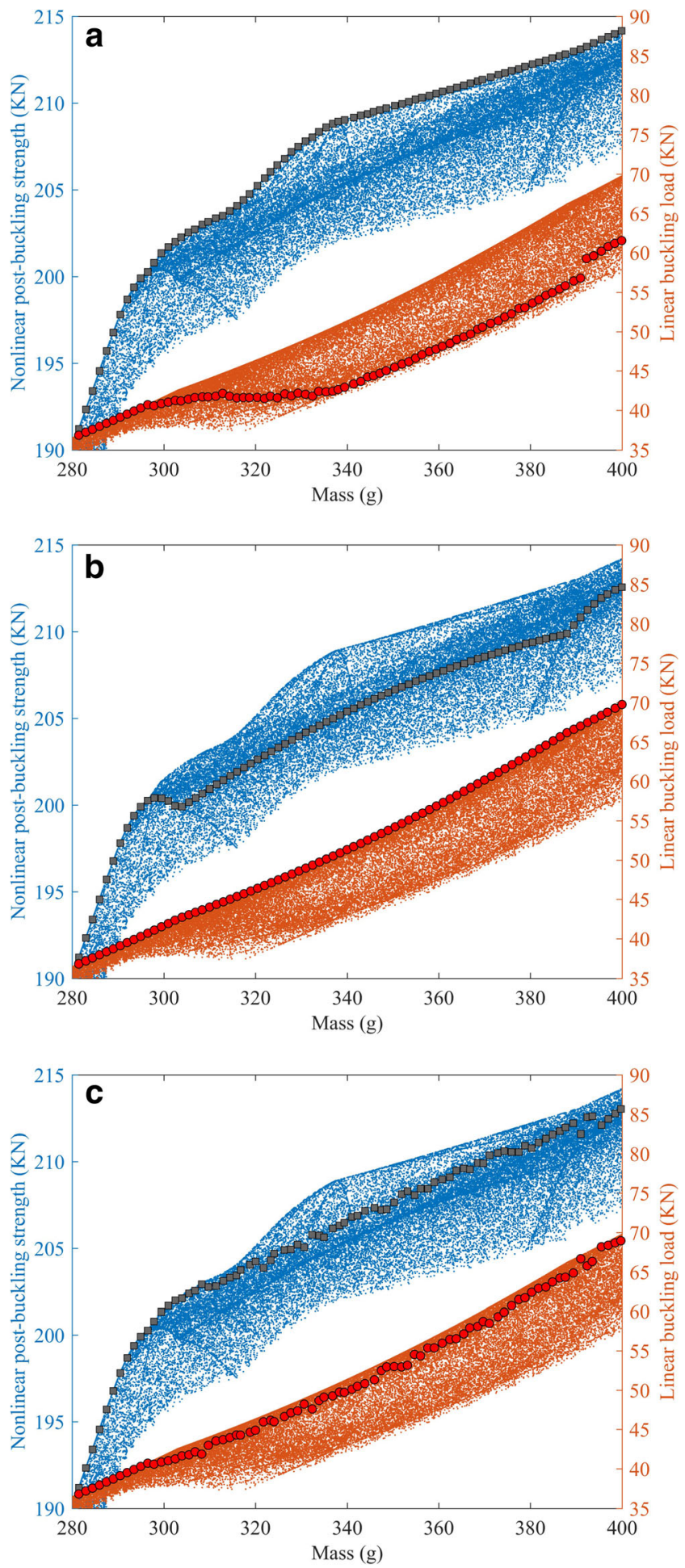

Fig. 4 Deterministic multi-objective optimisation results of the monostringer stiffened panel: a a large weight given to NPS objective; $\mathbf{b}$ a large weight given to LBL objective; $\mathbf{c}$ similar weights to both NPS and LBL objectives. For each case, squares and circles show the Pareto fronts of NPS and LBL, respectively

LBL. For the 2D diagram shown in Fig. 4, the squares and circles show the Pareto envelope for the NPS and LBL, respectively. Depending on the weight given to the LBL and NPS objectives in the optimisation algorithm, different Pareto fronts are obtained. Three Pareto envelopes are examined here and shown through sub-figures a-c showing the designs with (a) maximum nonlinear post-buckling strength, (b) maximum linear buckling load, and (c) maximum LBL and NPS. Figure $4 \mathrm{a}$ and $\mathrm{b}$ clearly show the competing nature of all objectives and specifically the competing objectives of maximising both LBL and NPS against minimising the mass. It is seen in sub-figure a that when a large weight is given NPS objective, the obtained optimised designs do not show an even near-optimum LBL. On the other hand, in sub-figure b, when a large weight is given to LBL, the reported optimised designs do not display near-optimum NPS. This is due the competing nature of these objectives where fully maximising one results in non-optimum value for the other one.

To examine the optimised omega-stringer geometries in more detail, Tables 3 and 4 are constructed showing the selection of points on the Pareto fronts corresponding to Fig. $4 \mathrm{~b}$ and a, respectively. More specifically, Table 3 shows the omega-stringer designs optimised for maximum linear buckling load and minimum mass.

It is interesting to note that the first parameter that is changed by the optimiser as the stringer mass is increased from $280 \mathrm{~g}$ is the flange. Additionally, it is seen that optimised designs for this case are associated with minimum height and angle and maximum flange size. Table 4 corresponds to Fig. $4 \mathrm{a}$, i.e. showing the stringer designs optimised for maximum nonlinear post-buckling strength and minimum mass. As seen in this table, optimised designs for NPS are associated with maximised flange size, similar to optimised designs for LBL; however, unlike the LBL optimised results, the optimised designs for maximum NPS tend to have near maximum values for height and angle parameters. Tables 3 and 4 clearly highlight the differences in designs of minimum-mass monostringer stiffened panels optimised for maximum LBL versus those optimised for maximum NPS.

Figure 5 shows the geometries of mono-stringer designs optimised for (a) maximum NPS, corresponding to a stringer design of $X_{1}=27.51 \mathrm{~mm}, X_{2}=30.0 \mathrm{~mm}, H=35.0 \mathrm{~mm}$ and $\theta=64.57^{\circ}$, and (b) maximum LBL, corresponding to a stringer design of $X_{1}=35.74 \mathrm{~mm}, X_{2}=30.0 \mathrm{~mm}, H=25.0 \mathrm{~mm}$ and $\theta=55.0^{\circ}$. The figure clearly shows the differences between the height and angle of the two stringer designs.

\section{Multi-objective probabilistic optimisation}

In this section, multi-objective probabilistic optimisations (MOPO) are conducted on the surrogate model developed in Section 3, while considering only the nonlinear post-buckling strength as an output of the surrogate model. RDO as well as RBDO is conducted. In particular, RDO is performed while considering the uncertainties in the stringer mechanical 
Table 3 Detailed optimisation results for case $b$ of the system of Fig. 4, i.e. stringer designs optimised for maximum LBL

\begin{tabular}{|c|c|c|c|c|c|c|}
\hline \multicolumn{4}{|c|}{ Stringer geometry (optimisation inputs) } & \multicolumn{3}{|c|}{ Optimisation objectives } \\
\hline $\begin{array}{l}\text { Flange, } X_{2} \\
(18-30 \mathrm{~mm})\end{array}$ & $\begin{array}{l}\text { Angle, } \theta \\
\left(55-65^{\circ}\right)\end{array}$ & $\begin{array}{l}\text { Foot, } X_{1} \\
(26-46 \mathrm{~mm})\end{array}$ & $\begin{array}{l}\text { Height, } H \\
(25-35 \mathrm{~mm})\end{array}$ & Stringer mass $(\mathrm{g})$ & NPS (KN) & $\mathrm{LBL}(\mathrm{KN})$ \\
\hline 19.74 & 55 & 26 & 25 & 281.45 & 191.21 & 36.83 \\
\hline 24.01 & 55 & 26 & 25 & 290.49 & 197.8 & 39.12 \\
\hline 28.25 & 55 & 26 & 25 & 299.49 & 200.4 & 41.53 \\
\hline 30 & 55 & 27.22 & 25 & 308.38 & 200.66 & 43.68 \\
\hline 30 & 55 & 29.37 & 25 & 317.49 & 202.15 & 45.73 \\
\hline 30 & 55 & 31.49 & 25 & 326.48 & 203.52 & 47.87 \\
\hline 30 & 55 & 33.62 & 25 & 335.49 & 204.79 & 50.14 \\
\hline 30 & 55 & 35.74 & 25 & 344.48 & 205.95 & 52.55 \\
\hline 30 & 55 & 37.86 & 25 & 353.48 & 206.98 & 55.1 \\
\hline 30 & 55 & 39.99 & 25 & 362.5 & 207.89 & 57.8 \\
\hline 30 & 55 & 42.11 & 25 & 371.49 & 208.69 & 60.63 \\
\hline 30 & 55 & 44.23 & 25 & 380.48 & 209.39 & 63.6 \\
\hline 30 & 55 & 46 & 25.29 & 389.49 & 210.39 & 66.6 \\
\hline 30 & 55 & 46 & 27.03 & 398.49 & 212.41 & 69.28 \\
\hline
\end{tabular}

properties as well as its geometric parameters. RBDO, on the other hand, is carried out while accounting only for uncertainties associated with mechanical properties. PESA-II multi-objective evolutionary optimisation algorithm is utilised for all the cases in this section.

\subsection{Robust-design optimisation}

In this section, two different RDOs are performed: one assuming uncertainties in mechanical properties, i.e. $E_{11}, E_{22}=E_{33}$,
$G_{23}$ and $G_{12}=G_{13}$, and the other considering uncertainties in stringer geometry, i.e. $X_{1}, X_{2}, H$ and $\theta$.

\subsubsection{RDO with uncertainties in mechanical properties}

The RDO conducted in this section assumes that the mechanical properties have a random normal distribution with $5 \%$ coefficient of variation (Akula 2014; Yang et al. 2013) and the mean values as given in Table 1. A direct Monte Carlo sampling approach is employed to conduct a robustness
Table 4 Detailed optimisation results for case a of the system of Fig. 4, i.e. stringer designs optimised for maximum NPS

\begin{tabular}{|c|c|c|c|c|c|c|}
\hline \multicolumn{4}{|c|}{ Stringer geometry (optimisation inputs) } & \multicolumn{3}{|c|}{ Optimisation objectives } \\
\hline $\begin{array}{l}\text { Flange, } X_{2} \\
(18-30 \mathrm{~mm})\end{array}$ & $\begin{array}{l}\text { Angle, } \theta \\
\left(55-65^{\circ}\right)\end{array}$ & $\begin{array}{l}\text { Foot, } X_{1} \\
(26-46 \mathrm{~mm})\end{array}$ & $\begin{array}{l}\text { Height, } H \\
(25-35 \mathrm{~mm})\end{array}$ & Stringer mass (g) & NPS $(\mathrm{KN})$ & $\mathrm{LBL}(\mathrm{KN})$ \\
\hline 19.74 & 55 & 26 & 25 & 281.45 & 191.21 & 36.83 \\
\hline 24.01 & 55 & 26 & 25 & 290.49 & 197.8 & 39.12 \\
\hline 30 & 57.51 & 26 & 25 & 299.47 & 201.35 & 40.85 \\
\hline 30 & 58.66 & 26 & 27.12 & 308.42 & 202.99 & 41.71 \\
\hline 30 & 61.55 & 26 & 29.77 & 317.36 & 204.41 & 41.61 \\
\hline 30 & 63.05 & 26.02 & 32.06 & 326.4 & 206.8 & 42.09 \\
\hline 30 & 64.8 & 26 & 34.49 & 335.42 & 208.62 & 42.34 \\
\hline 30 & 64.57 & 27.51 & 35 & 344.49 & 209.4 & 44.24 \\
\hline 30 & 64.65 & 29.65 & 35 & 353.48 & 210.09 & 46.19 \\
\hline 29.97 & 64.32 & 31.69 & 35 & 362.46 & 210.77 & 48.51 \\
\hline 30 & 63.85 & 33.6 & 35 & 371.33 & 211.49 & 51.05 \\
\hline 30 & 63.66 & 35.69 & 35 & 380.46 & 212.24 & 53.63 \\
\hline 30 & 63.31 & 37.69 & 35 & 389.44 & 212.97 & 56.44 \\
\hline 24.89 & 59.43 & 40.89 & 35 & 398.39 & 214 & 61.19 \\
\hline
\end{tabular}


(a)

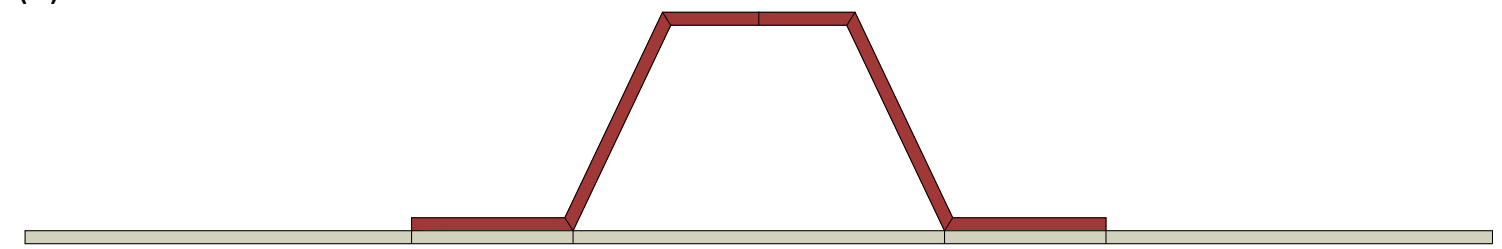

(b)

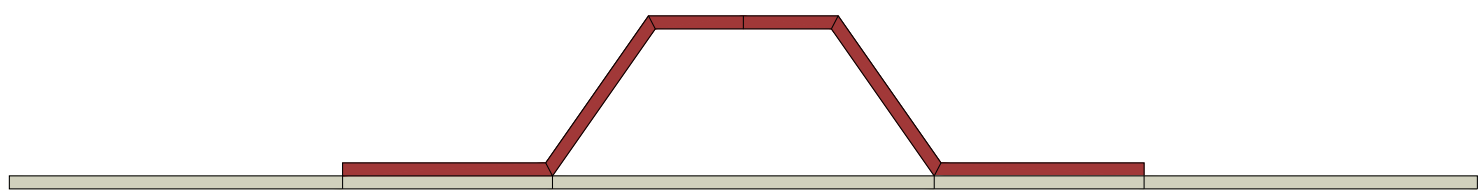

Fig. 5 Mono-stringer stiffened panel geometries optimised for minimum mass and a maximum nonlinear post-buckling strength and $\mathbf{b}$ maximum linear buckling load. The mass of the stringer for both cases is around $344.5 \mathrm{~g}$

analysis within the optimisation loop. To ensure the accuracy of the Monte Carlo approach, a random normal distribution consisting of $10^{5}$ samples is considered for each of the mechanical properties, as shown in Fig. 6.

More specifically, each time the objective function is called by the optimiser, a Monte Carlo loop consisting of $10^{5}$ runs is

\section{(a)}

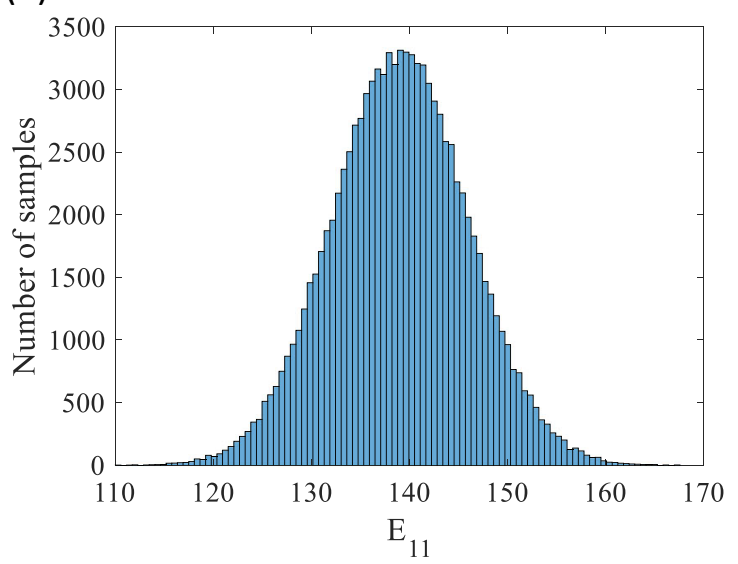

(c)

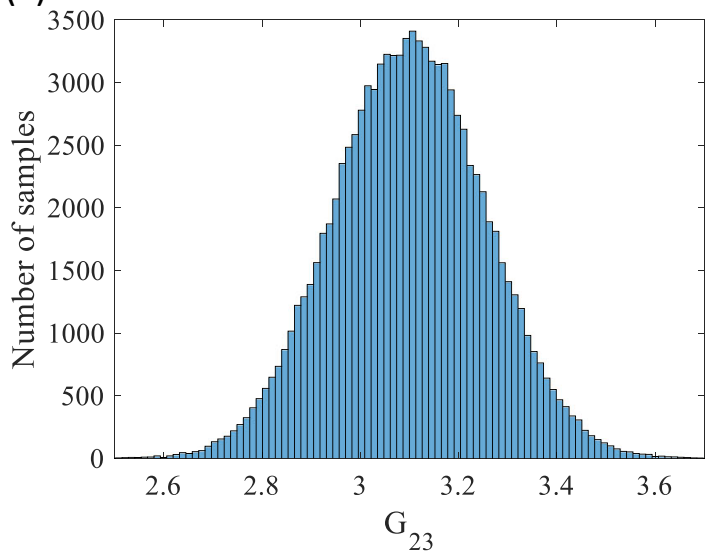

performed and the mean value and standard deviation of the output, i.e. NPS, are passed to the optimiser. The optimiser objectives are minimising mass, maximising NPS mean value and minimising the standard deviation of NPS. Given that there are three objectives to be optimised, a population size of 100 is selected with a maximum of 100 iterations to ensure

(b)

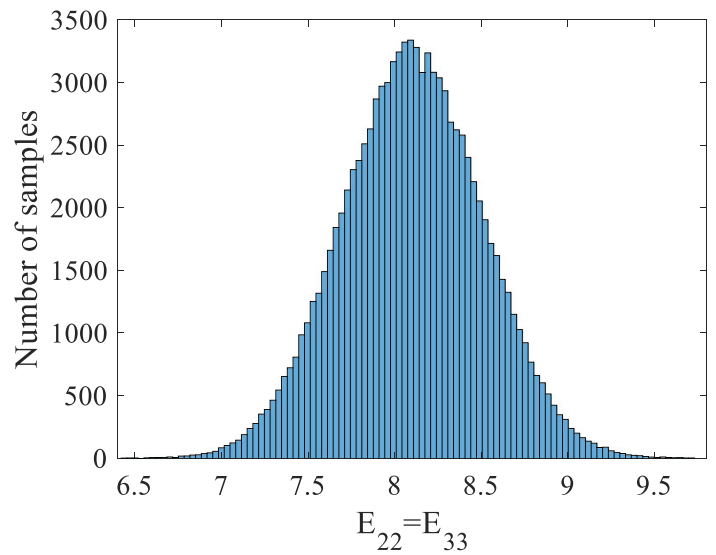

(d)

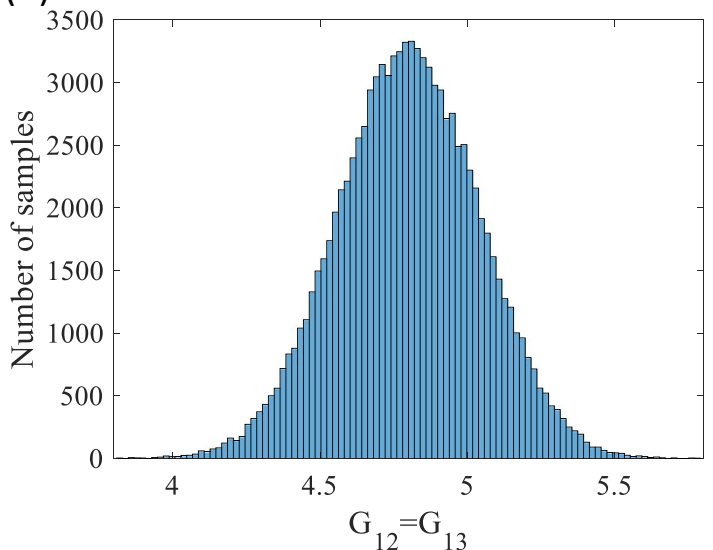

Fig. 6 a-d Random normal distributions of the mechanical properties of the mono-stringer stiffened panel considering a coefficient of variation of 5\% and $10^{5}$ samples 
that all optimised solutions in the design space are found. This results in $10^{4}$ runs in the optimisation loop and a total of $10^{9}$ runs in the Monte Carlo-nested optimisation algorithm. This large number of runs requires the surrogate model to be optimised for very fast run time. It is worth noting that even a surrogate model run time of $1 \mathrm{~ms}$ results in $278 \mathrm{~h}$ (11 days!) total run time for the RDO to be completed. In this study, a $20 \times$ speed up is achieved via developing a vectorised surrogate model as well as taking advantage of parallel computing.

In order to better highlight the differences between robust and deterministic designs of the mono-stringer panel, three cases are considered and the corresponding optimisation results are plotted in Fig. 7 through sub-figures a-c. As mentioned before, the first, second and third objectives are minimising mass, maximising NPS mean value and minimising NPS standard deviation. For the first case, shown in sub-figure a, a large weight is given to the second optimisation objective, i.e. NPS mean value. This will result in stringer designs which are very similar to those obtained via deterministic optimisation; the purpose of this figure is to examine the robustness of deterministic designs in the presence of uncertainties in mechanical properties. The next two cases correspond respectively to RDOs with weights 5 (RDO-1) and 10 (RDO-2) given to the NPS standard deviation and weight 1 given to the other two objectives. The results of RDO-1 and RDO-2 are plotted in sub-figures $b$ and c, respectively. Figure 7 clearly shows the trend that reduced NPS standard deviation comes at the price of a reduction in NPS mean value. However, comparing sub-figures $a$ and $b$ shows that the RDO1 approach manages to reduce the NPS standard deviation significantly without reducing the NPS mean value by much. The reduction in the NPS mean value is more for the case RDO-2 in order to achieve narrower NPS distributions, i.e. smaller NPS standard deviations.

In order to be able to compare the stringer geometries corresponding to each case, Table 5 is constructed, listing a summary of the Pareto fronts of cases RDO-1 and RDO-2, corresponding to Fig. $7 \mathrm{~b}$ and c, respectively. It should be noted that the stringer geometries corresponding to case 1, i.e. Fig. 7a, are almost identical to those of the deterministic optimisation given in Table 4 . Comparing the results of RDO-1 to those of DO shows that for stringer masses larger than $319 \mathrm{~g}$, the optimised stringer geometries based on RDO-1 have maximum possible angle $\left(65^{\circ}\right)$, while those based on DO have angles varying from $63^{\circ}$ to $65^{\circ}$. A comparison of RDO-2 results with the other cases reveals that the optimised geometries based on RDO-2 have very different angles and heights compared to RDO-1 and DO results. In particular, for a large spectrum of mass, the angle and height vary in the vicinity of $58^{\circ}$ and $26 \mathrm{~mm}$, as opposed to $63-65^{\circ}$ and $35 \mathrm{~mm}$ of the other two cases. For stringer masses more than $390 \mathrm{~g}$, both RDOs result in the same stringer geometries while DO results in stringer geometries with reduced angle and flange size. In order to better illustrate the differences between robust
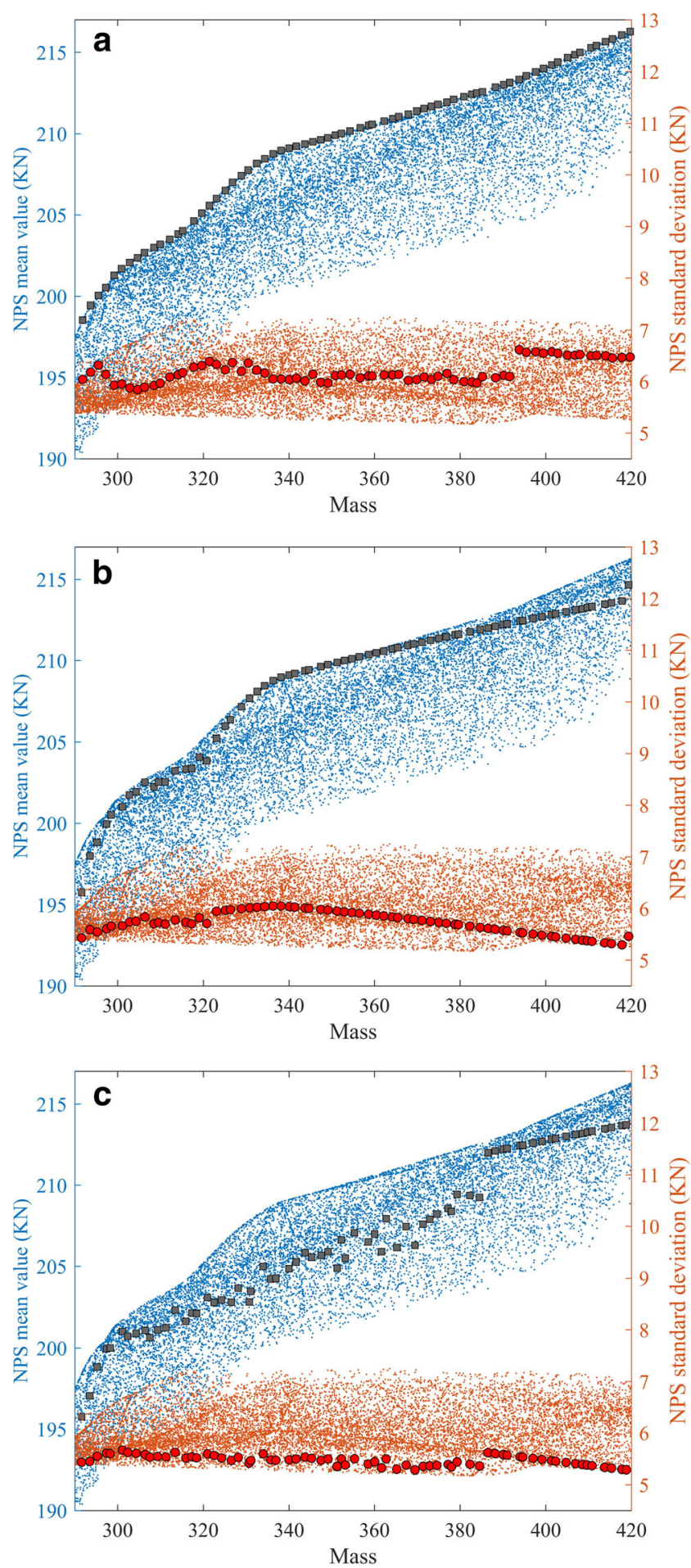

Fig. 7 Multi-objective robust-design optimisation results of the monostringer stiffened panel: a a large weight given to NPS mean value objective; $\mathbf{b}$, $\mathbf{c}$ increased weights of NPS standard deviation objective. For each case, squares and circles show the Pareto fronts of NPS mean value and standard deviation, respectively

and deterministic designs, the mono-stringer designs obtained based on the two approaches are plotted in Fig. 8 for a stringer of mass $399 \mathrm{~g}$. The deterministic design corresponds to a stringer of $X_{1}=41.04 \mathrm{~mm}, X_{2}=25.03 \mathrm{~mm}$, 
Table 5 Detailed optimisation results for cases a and b of the system of Fig. 7, denoted by RDO-1 and RDO-2, respectively

\begin{tabular}{|c|c|c|c|c|c|c|c|}
\hline & \multicolumn{4}{|c|}{ Stringer geometry (optimisation inputs) } & \multicolumn{3}{|c|}{ Optimisation objectives } \\
\hline & Flange, $X_{2}(18-30 \mathrm{~mm})$ & Angle, $\theta\left(55-65^{\circ}\right)$ & Foot, $X_{1}(26-46 \mathrm{~mm})$ & Height, $H(25-35 \mathrm{~mm})$ & $\begin{array}{l}\text { Stringer } \\
\text { mass }(\mathrm{g})\end{array}$ & NPS mean $(\mathrm{KN})$ & NPS STD $(\mathrm{KN})$ \\
\hline \multirow[t]{14}{*}{ RDO-1 } & 27.39 & 59.25 & 26 & 25 & 291.6 & 195.76 & 5.43 \\
\hline & 30 & 59.34 & 26 & 25.83 & 301.13 & 201.01 & 5.67 \\
\hline & 30 & 58.77 & 27.48 & 26.12 & 309.57 & 202.54 & 5.73 \\
\hline & 30 & 58.56 & 28.86 & 26.8 & 319.07 & 204.07 & 5.82 \\
\hline & 30 & 65 & 26 & 33.16 & 328.94 & 207.17 & 6 \\
\hline & 30 & 65 & 26.15 & 35 & 338.18 & 208.97 & 6.05 \\
\hline & 30 & 65 & 28.34 & 35 & 347.46 & 209.63 & 5.98 \\
\hline & 30 & 65 & 30.47 & 35 & 356.48 & 210.23 & 5.91 \\
\hline & 30 & 65 & 32.59 & 35 & 365.45 & 210.79 & 5.83 \\
\hline & 30 & 65 & 34.85 & 35 & 375.05 & 211.36 & 5.73 \\
\hline & 30 & 65 & 37.12 & 35 & 384.69 & 211.91 & 5.63 \\
\hline & 30 & 65 & 39.32 & 35 & 393.99 & 212.43 & 5.54 \\
\hline & 30 & 65 & 41.28 & 35 & 402.3 & 212.87 & 5.45 \\
\hline & 30 & 65 & 43.29 & 35 & 410.81 & 213.32 & 5.36 \\
\hline \multirow[t]{14}{*}{ RDO-2 } & 27.39 & 59.25 & 26 & 25 & 291.6 & 195.76 & 5.43 \\
\hline & 30 & 59.34 & 26 & 25.83 & 301.13 & 201.01 & 5.67 \\
\hline & 29.07 & 58.87 & 28.35 & 25.75 & 309.35 & 201.1 & 5.54 \\
\hline & 29.1 & 58.54 & 30.44 & 25.7 & 318.44 & 202.11 & 5.52 \\
\hline & 29.11 & 58.7 & 31.9 & 26.45 & 328.18 & 203.67 & 5.52 \\
\hline & 30 & 58.61 & 34.29 & 25.78 & 336.97 & 204.26 & 5.47 \\
\hline & 30 & 58.15 & 36.61 & 25.8 & 347.54 & 205.67 & 5.47 \\
\hline & 29.55 & 58.36 & 37.42 & 26.92 & 355.34 & 207.07 & 5.5 \\
\hline & 29.23 & 58.49 & 40.62 & 26.35 & 365.2 & 206.16 & 5.3 \\
\hline & 30 & 58.38 & 42.04 & 26.67 & 374.57 & 208.21 & 5.37 \\
\hline & 29.6 & 58.01 & 44.28 & 26.82 & 384.54 & 209.25 & 5.36 \\
\hline & 30 & 65 & 39.32 & 35 & 393.99 & 212.43 & 5.54 \\
\hline & 30 & 65 & 41.28 & 35 & 402.3 & 212.87 & 5.45 \\
\hline & 30 & 65 & 43.29 & 35 & 410.81 & 213.32 & 5.36 \\
\hline
\end{tabular}

$H=35.0 \mathrm{~mm}$ and $\theta=59.39^{\circ}$, while the robust one corresponds to a stringer of $X_{1}=40.53 \mathrm{~mm}, X_{2}=30.0 \mathrm{~mm}, H=$ $35.0 \mathrm{~mm}$ and $\theta=65.0^{\circ}$. It is clearly seen in the figure that the robust design has a larger angle as well as a larger flange. The probability density function (PSD) plots of the two designs are depicted in Fig. 9, showing that the robust design has a slightly smaller NPS mean value, accompanied by a narrower distribution.

\subsubsection{RDO with uncertainties in stringer geometry}

The RDO conducted in this section aims at minimising mass and NPS standard deviation and maximising NPS mean value while considering uncertainties in the stringer geometry. The geometric inputs are assumed to have a truncated random normal distribution with $0.1 \%$ coefficient of variation; the mean values are determined by the optimiser each time the objective

(a)

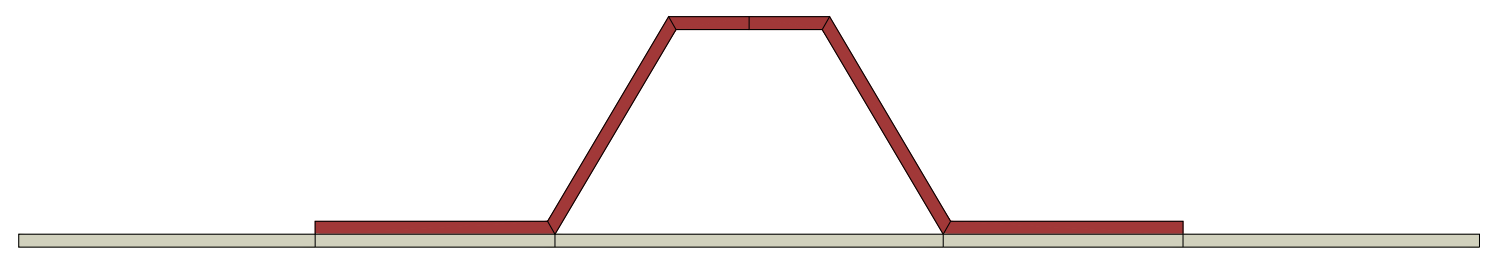

(b)

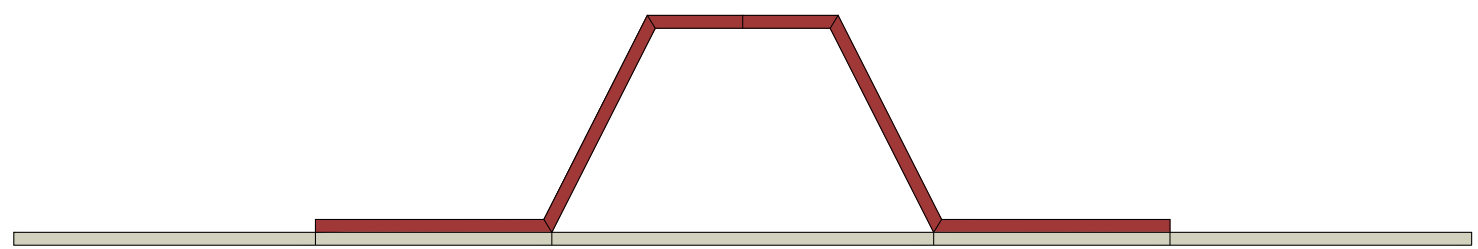

Fig. 8 Mono-stringer stiffened panel geometry optimised for maximum nonlinear post-buckling strength based on: a deterministic optimisation and $\mathbf{b}$ robust-design optimisation (uncertainties in mechanical properties). The mass of the stringer for both cases is around $399 \mathrm{~g}$ 
Fig. 9 Probability density function plot of NPS distribution for the deterministic and robust mono-stringer designs given in Fig. 6

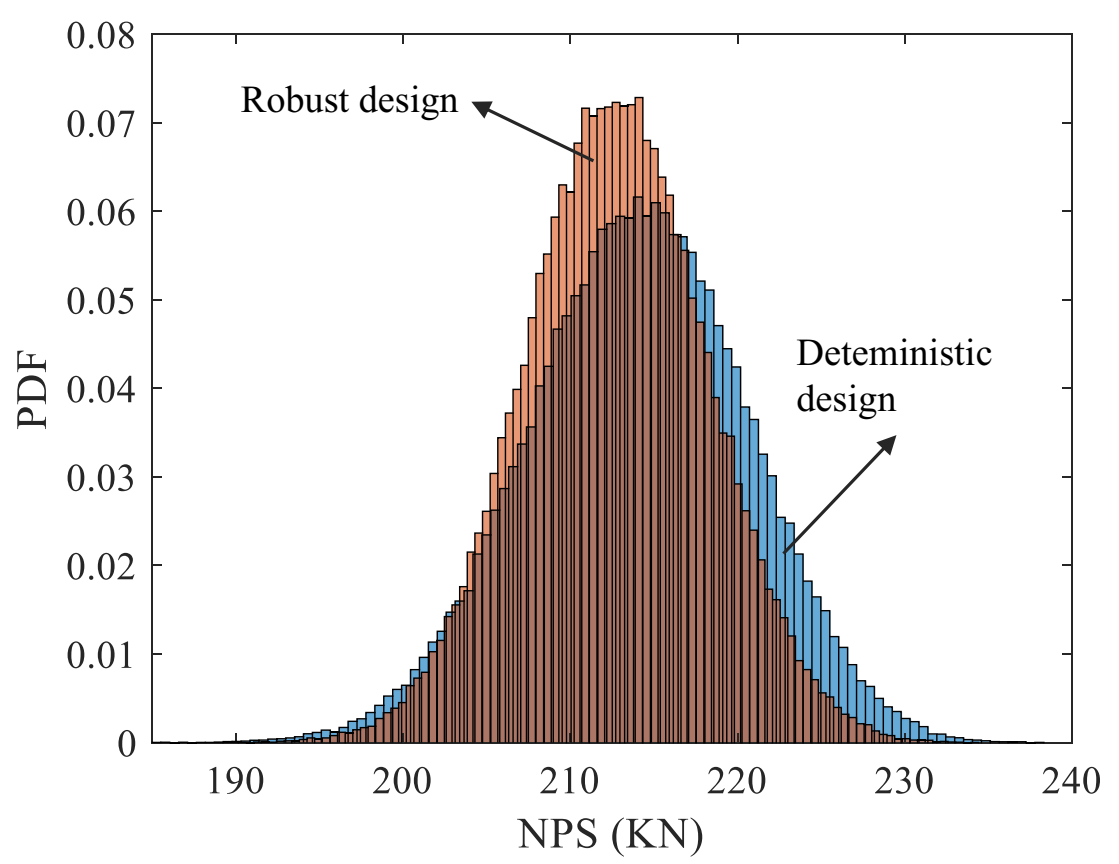

(a)

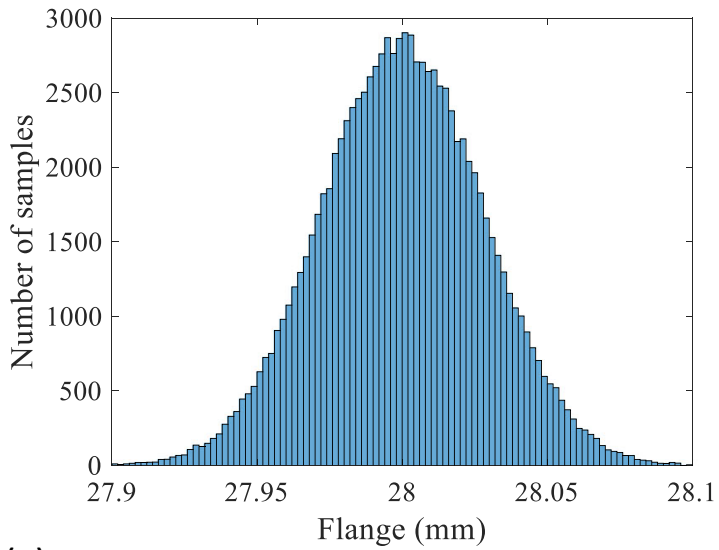

(c)

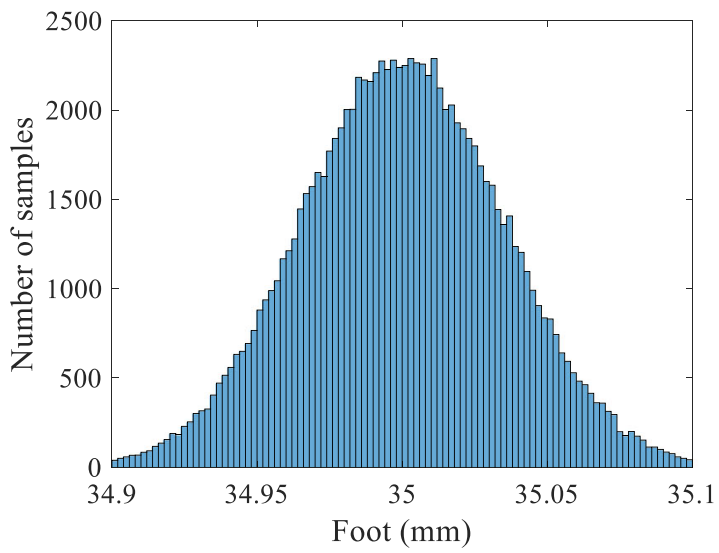

(b)

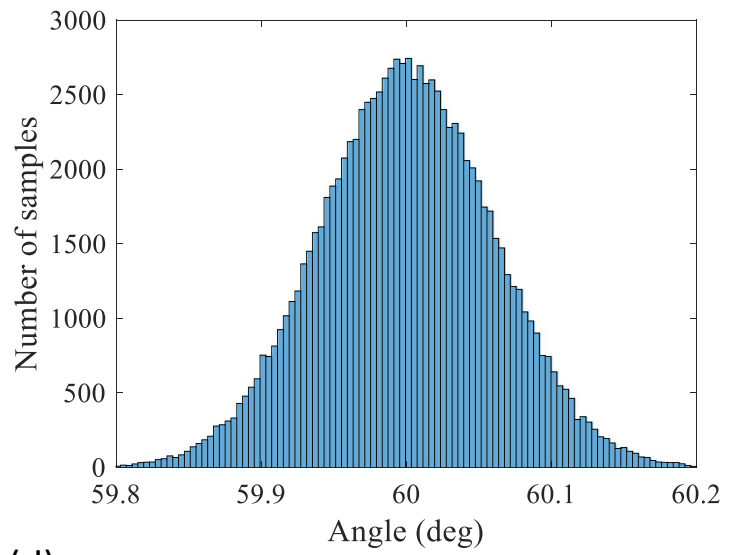

(d)

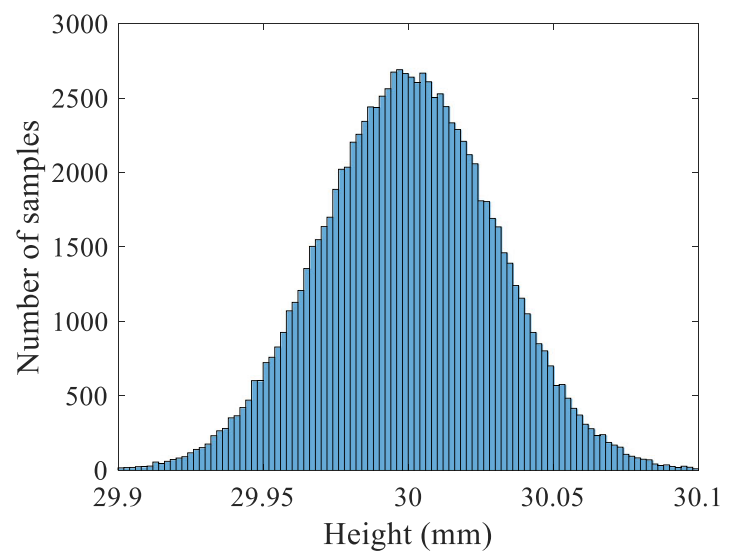

Fig. 10 a-d Examples of truncated random normal distributions of the stringer geometric parameters considering a coefficient of variation of $0.1 \%$ and manufacturing tolerances of $\pm 0.1 \mathrm{~mm}$ for flange, foot and height and $\pm 0.2^{\circ}$ for angle 

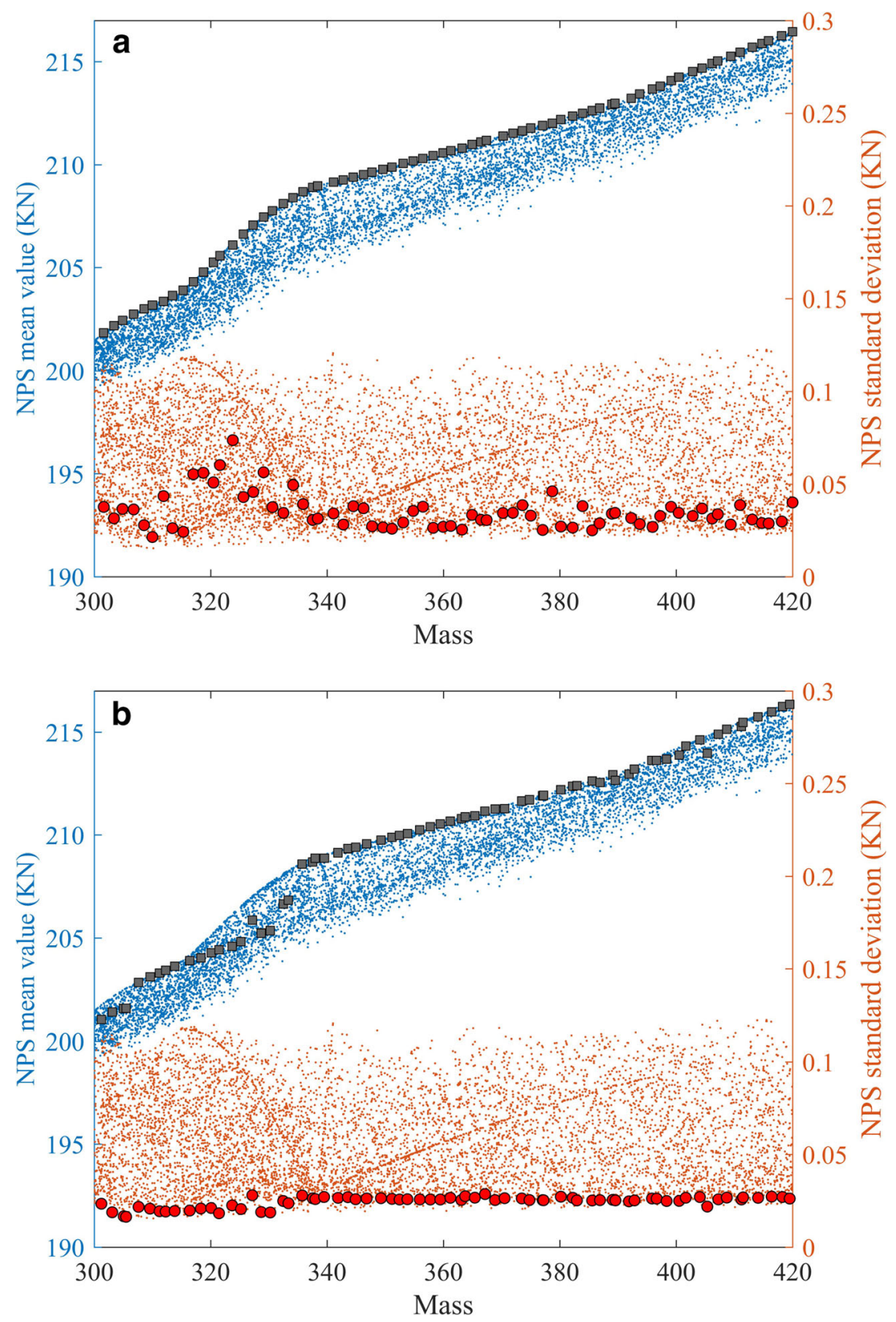

Fig. 11 Multi-objective robust-design optimisation results of the monostringer stiffened panel: a a large weight given to NPS mean value objective; $\mathbf{b}$ a large weight given to NPS standard deviation objective.

function is called. Similar to the previous case, a direct Monte Carlo sampling approach is implemented in the objective function to conduct a robustness analysis within the optimisation loop. A truncated random normal distribution consisting of $10^{5}$ samples is considered for each geometric input with manufacturing tolerances of $\pm 0.1 \mathrm{~mm}$ for $X_{1}, X_{2}$ and $H$ and $\pm 0.2^{\circ}$ for $\theta$. An example of the truncated random normal distribution for each of the inputs is shown in Fig. 10, for a stringer with mean geometric inputs of $X_{1}=35.0 \mathrm{~mm}, X_{2}=28.0 \mathrm{~mm}$, $H=30.0 \mathrm{~mm}$ and $\theta=60.0^{\circ}$. Similar to the previous case,
For each case, squares and circles show the Pareto fronts of NPS mean value and standard deviation, respectively

PESA-II optimisation algorithm is used with a population size of 100 and a maximum of 100 iterations.

The results of the RDO with uncertainties in geometric parameters are plotted in Fig. 11. Sub-figure a corresponds to an RDO with a large weight given to the NPS mean value objective, while for the case in sub-figure $b$, a large weight is given to NPS standard deviation objective. As explained before, when a large weight is given to NPS mean value, the optimisation results become very similar to deterministic optimisation with only two objectives of minimising mass and maximising NPS. 
Table 6 Detailed optimisation results for case b of the system of Fig. 11

\begin{tabular}{|c|c|c|c|c|c|c|}
\hline \multicolumn{4}{|c|}{ Stringer geometry (optimisation inputs) } & \multicolumn{3}{|c|}{ Optimisation objectives } \\
\hline Flange, $X_{2}(18-30 \mathrm{~mm})$ & Angle, $\theta\left(55-65^{\circ}\right)$ & Foot, $X_{1}(26-46 \mathrm{~mm})$ & Height, $H(25-35 \mathrm{~mm})$ & Stringer mass (g) & NPS mean $(\mathrm{KN})$ & NPS STD $(\mathrm{KN})$ \\
\hline 26.87 & 55 & 26 & 25.9 & 301.23 & 201.03 & 0.0233 \\
\hline 29.95 & 58.65 & 26 & 27.37 & 309.58 & 203.12 & 0.0206 \\
\hline 30 & 58.78 & 27.18 & 28.14 & 318.32 & 204.04 & 0.0207 \\
\hline 26.98 & 61.49 & 26 & 33.11 & 327.16 & 205.87 & 0.0279 \\
\hline 30 & 64.31 & 26 & 34.42 & 335.74 & 208.59 & 0.0278 \\
\hline 30 & 64.62 & 27.31 & 35 & 343.58 & 209.33 & 0.0268 \\
\hline 30 & 64.2 & 29.26 & 35 & 352.47 & 209.97 & 0.0256 \\
\hline 30 & 64.06 & 31.28 & 35 & 361.2 & 210.68 & 0.0265 \\
\hline 30 & 63.71 & 32.97 & 35 & 368.88 & 211.26 & 0.0253 \\
\hline 30 & 63.44 & 34.85 & 35 & 377.2 & 211.92 & 0.0252 \\
\hline 29.61 & 62.79 & 37.12 & 35 & 386.91 & 212.55 & 0.0252 \\
\hline 25.23 & 59.4 & 40.1 & 35 & 395.84 & 213.62 & 0.0261 \\
\hline 25.08 & 59.17 & 42.01 & 35 & 404.07 & 214.62 & 0.0269 \\
\hline 25.04 & 58.89 & 43.67 & 35 & 411.5 & 215.47 & 0.0267 \\
\hline
\end{tabular}

The goal of constructing Fig. 11a is to measure the robustness of the stringer designs with maximum possible NPS mean value, while Fig. $11 \mathrm{~b}$ demonstrates the amount of reduction in NPS mean value as a result of minimising the NPS standard deviation. As seen in Fig. 11b, for a large portion of the mass spectrum, the NPS mean value does not change much compared to Fig. 11a while NPS standard deviation is minimised. To show the changes in stringer geometry corresponding to Fig. 11b, Table 6 is constructed showing the optimisation inputs and outputs for a wide range of stringer masses. Comparing these optimised designs to those of the deterministic optimisation shows that the stringer angle and foot have been changed slightly to accommodate more robust designs.

A comparison between the deterministic $\left(X_{1}=26.0 \mathrm{~mm}, X_{2}=\right.$ $30.0 \mathrm{~mm}, H=31.91 \mathrm{~mm}$ and $\left.\theta=63.0^{\circ}\right)$ and robust $\left(X_{1}=\right.$
$28.83 \mathrm{~mm}, X_{2}=29.82 \mathrm{~mm}, H=28.06 \mathrm{~mm}$ and $\left.\theta=58.33^{\circ}\right)$ designs is shown in Fig. 12 for a stringer of mass $325 \mathrm{~g}$; the PDF plots of the NPS for the two designs are shown in Fig. 13. Another comparison between NPS distribution of deterministic and robust stringer designs of mass $378.7 \mathrm{~g}$ is shown in Fig. 14, with the robust stringer design being of $X_{1}=35.46 \mathrm{~mm}, X_{2}=28.85 \mathrm{~mm}$, $H=35.0 \mathrm{~mm}$ and $\theta=62.48^{\circ}$ and the deterministic one being of $X_{1}=35.45 \mathrm{~mm}, X_{2}=30.0 \mathrm{~mm}, H=35.0 \mathrm{~mm}$ and $\theta=64.17^{\circ}$.

\subsection{Reliability-based design optimisation}

In this section, a reliability-based design optimisation is conducted by considering uncertainties in the mechanical properties, i.e. $E_{11}, E_{22}=E_{33}, G_{23}$ and $G_{12}=G_{13}$. The RBDO process ensures that the final design meets a certain

(a)

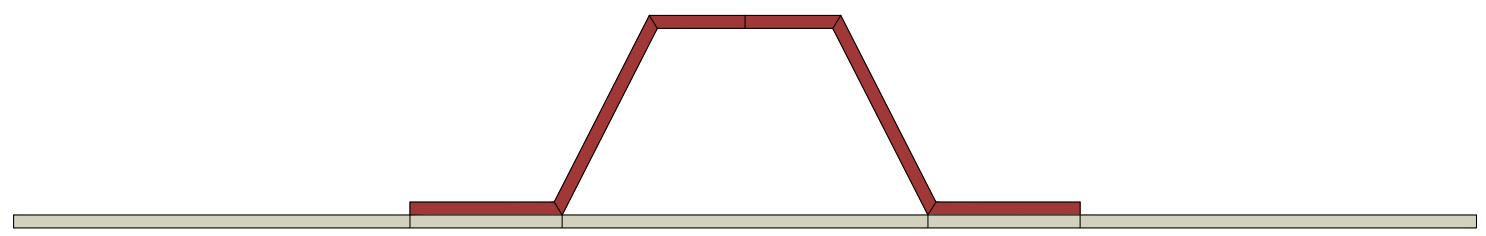

(b)

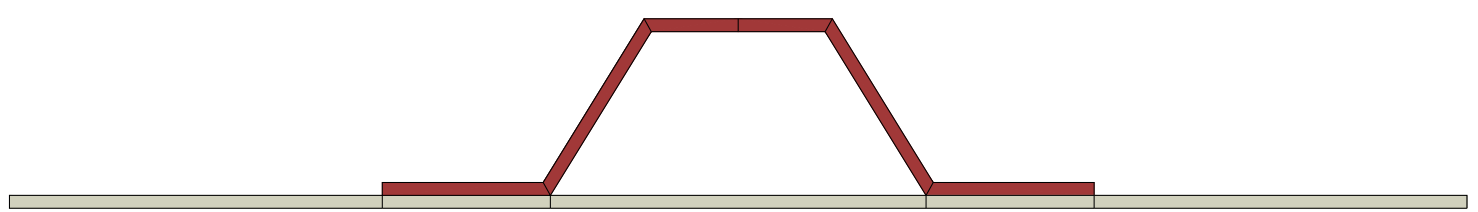

Fig. 12 Mono-stringer stiffened panel geometry optimised for maximum nonlinear post-buckling strength based on: a deterministic optimisation and $\mathbf{b}$ robust-design optimisation (geometric uncertainties). The mass of the stringer for both cases is around $325 \mathrm{~g}$ 
Fig. 13 Probability density function plot of NPS distribution for the robust and deterministic mono-stringer designs given in Fig. 10

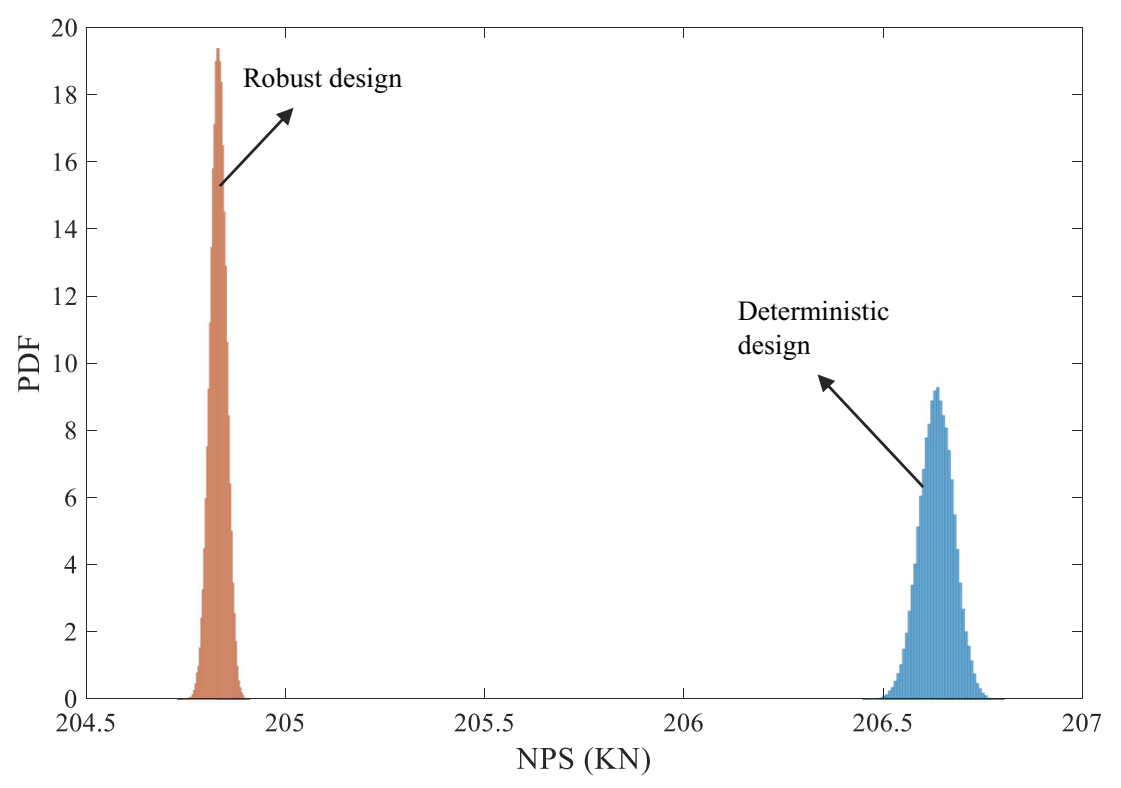

probabilistic constraint up to a prescribed reliability level $\beta_{t}$ as explained in Section 2.4. The RBDO is performed by including a reliability analysis within the optimisation loop. More specifically, a reliability analysis algorithm based on the firstorder reliability method (FORM) is formulated within the objective function to calculate the reliable NPS based on a prescribed reliability index $\beta_{t}$. The reliability analysis is solved through use of a hybrid mean value (HMV) method (Youn et al. 2003).

The reliability analysis technique employed in this study is validated using direct Monte Carlo sampling, which is commonly used as benchmark for validation purposes. Although direct Monte Carlo sampling is the most accurate method for reliability analysis, it becomes very time consuming for large reliability indices. The comparison is conducted for a stringer of $X_{1}=35.0 \mathrm{~mm}, X_{2}=25.0 \mathrm{~mm}, H=30.0 \mathrm{~mm}$ and $\theta=60.0^{\circ}$, for reliability indices 3 and 4 . For each reliability index, the corresponding NPS is obtained via the reliability analysis technique employed in this study (HMV) and direct Monte Carlo sampling. Three Monte Carlo sampling cases are considered with $10^{5}, 10^{6}$ and $10^{7}$ samples, to ensure converged result. Due to the stochastic nature of the Monte Carlo simulations, for each case, the average of 5 different runs is reported as the final probabilistic NPS. The results of the comparison are shown in Table 7.
Fig. 14 Probability density function plot of NPS distribution for the robust and deterministic mono-stringer designs; mass of the stringer for both cases is around $378.7 \mathrm{~g}$

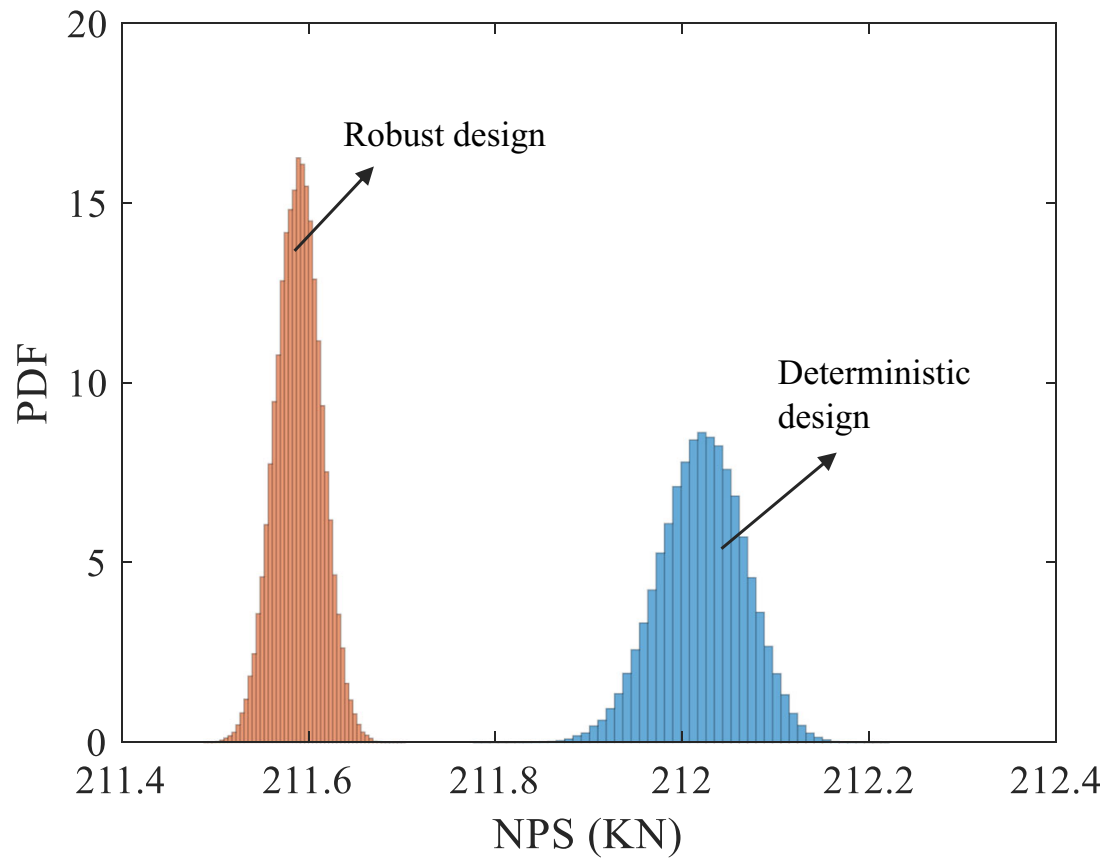


Table 7 Comparison of the reliability analysis results obtained via the method employed in this study (HMV) and those obtained using direct Monte Carlo sampling

\begin{tabular}{llllll}
\hline Prescribed reliability & \multicolumn{2}{l}{ NPS $(\mathrm{KN})$} & \% error \\
\cline { 2 - 5 } & HMV method & \multicolumn{2}{l}{ Monte Carlo (average of 5 runs) } & \\
\cline { 3 - 5 } & & $10^{5}$ samples & $10^{6}$ samples & $10^{7}$ samples & \\
\hline$\beta_{t}=3.0$ & 185.25 & 185.53 & 185.40 & 185.40 & 0.08 \\
$\beta_{t}=4.0$ & 178.27 & 178.09 & 178.32 & 178.38 & 0.06 \\
\hline
\end{tabular}

As seen, for both $\beta_{t}=3.0$ and 4.0 , the results of the reliability analysis technique employed in this study are very close to those obtained via the direct Monte Carlo sampling technique verifying the accuracy of the employed method.
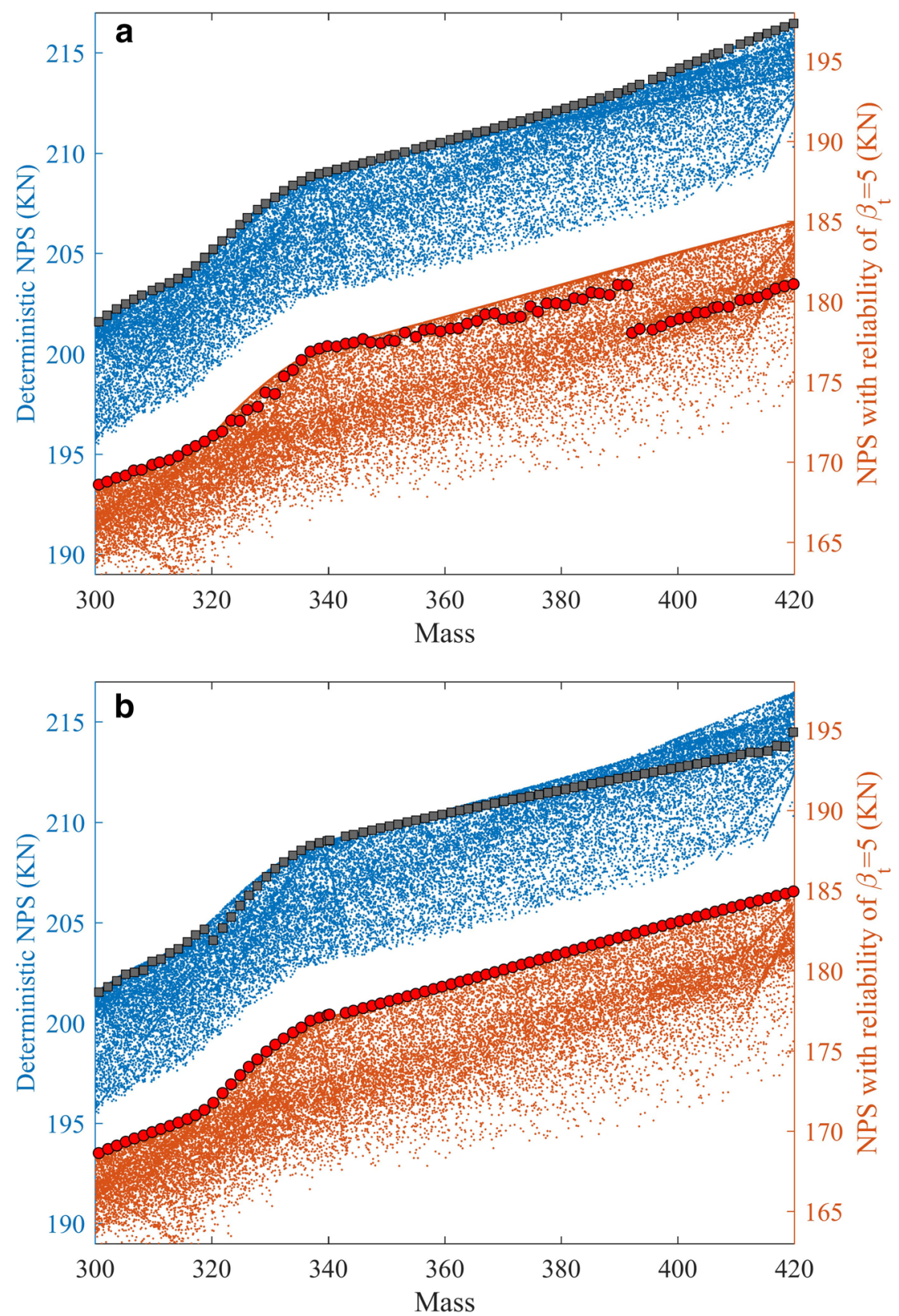

Fig. 15 Multi-objective reliability-based design optimisation results of the mono-stringer stiffened panel: a a large weight given to deterministic NPS objective; b a large weight given to NPS with reliability of $\beta=5.0$. For each case, squares and circles show the Pareto fronts of deterministic and reliable NPS, respectively 
Table 8 Detailed optimisation results for case b of the system of Fig. 15

\begin{tabular}{|c|c|c|c|c|c|c|}
\hline \multicolumn{4}{|c|}{ Stringer geometry (optimisation inputs) } & \multicolumn{3}{|c|}{ Optimisation objectives } \\
\hline Flange, $X_{2}(18-30 \mathrm{~mm})$ & Angle, $\theta\left(55-65^{\circ}\right)$ & Foot, $X_{1}(26-46 \mathrm{~mm})$ & Height, $H(25-35 \mathrm{~mm})$ & Stringer mass (g) & NPS $(\mathrm{KN})$ & $\mathrm{NPS}_{\beta t=5.0}(\mathrm{KN})$ \\
\hline 30 & 58.22 & 26 & 25.43 & 300.63 & 201.52 & 168.64 \\
\hline 30 & 59.68 & 26 & 27.69 & 309.78 & 203.07 & 169.95 \\
\hline 30 & 62.95 & 26 & 30.45 & 318.77 & 204.68 & 171.33 \\
\hline 30 & 65 & 26 & 32.93 & 327.83 & 206.82 & 174.48 \\
\hline 30 & 65 & 26 & 34.88 & 336.96 & 208.83 & 176.89 \\
\hline 30 & 65 & 28 & 35 & 346 & 209.51 & 177.67 \\
\hline 30 & 65 & 30.13 & 35 & 355.04 & 210.11 & 178.56 \\
\hline 30 & 65 & 32.26 & 35 & 364.05 & 210.67 & 179.46 \\
\hline 30 & 65 & 34.41 & 35 & 373.2 & 211.22 & 180.39 \\
\hline 30 & 65 & 36.55 & 35 & 382.25 & 211.75 & 181.3 \\
\hline 30 & 65 & 38.68 & 35 & 391.28 & 212.26 & 182.21 \\
\hline 30 & 65 & 40.82 & 35 & 400.36 & 212.76 & 183.1 \\
\hline 30 & 65 & 42.94 & 35 & 409.33 & 213.24 & 183.95 \\
\hline 30 & 64.95 & 45.08 & 35 & 418.49 & 213.78 & 184.79 \\
\hline
\end{tabular}

The PESA-II optimisation algorithm is employed with a population size of 300 and a maximum of 100 iterations. The goal of an RBDO is to maximise/minimise an objective while meeting a certain probabilistic constraint up to a prescribed reliability level. In this study, the ultimate nonlinear post-buckling strength, denoted as NPS, is considered in both the objective function and in the probabilistic constraint. In other words, the optimiser aims at maximizing the NPS while ensuring that the reliability is larger than the prescribed value.

Since that the mass of the stringer is an optimisation objective as well, selecting a specific value for the NPS to measure the reliability from does not serve the purpose, as the maximum NPS is varying with mass. Instead, three objectives are considered and optimised, i.e. the mass of the stringer, to be minimised; deterministic NPS, to be maximised; and the NPS with the prescribed reliability of $\beta_{t}$, to be maximised. Hence, for a given reliability index, the optimisation algorithm finds the minimum-mass designs which are optimised for not only the maximum deterministic NPS, but also the maximum reliable NPS. The results of this multi-objective probabilistic optimisation are shown in Fig. 15 through sub-figures a and b. Sub-figure a corresponds to an optimisation with a large weight given to the deterministic NPS objective, while sub-figure b shows the results for a case with a large weight given to reliable NPS. The optimisation is conducted assuming a reliability index of $\beta_{t}=5$. As seen in Fig. 15a, the designs optimised for maximum deterministic NPS are not necessarily the most reliable designs especially when the mass is larger than $390 \mathrm{~g}$. Figure $15 \mathrm{~b}$ shows that the optimum designs having maximum NPS up to the desired reliability level of $\beta_{t}=5.0$ can be achieved without reducing the maximum deterministic NPS significantly. The detailed optimisation results for case b of Fig. 15 are listed in Table 8.
The PDF plots of the NPS distribution associated with deterministic and reliable stringer designs of masses 368.5 and $391.5 \mathrm{~g}$ are depicted in Fig. 16 using $10^{6}$ points with the solid and dashed vertical lines showing the NPS with a reliability of $\beta_{t}=5.0$ for reliable and deterministic designs, respectively. As seen, the reliable designs for both cases have slightly smaller NPS mean value, but visibly larger NPS with desired reliability level of $\beta_{t}=5.0$.

\section{Conclusions}

In this study, a multi-objective design optimisation approach is presented for probabilistic analysis of composite structures. The approach is demonstrated on a mono-stringer stiffened panel for robust and reliability-based design optimisations. In particular, an accurate and comprehensive surrogate model is developed with the stringer geometry and the composite material mechanical properties as the inputs and the fundamental linear buckling load and the nonlinear post-buckling strength as the outputs. Multiobjective probabilistic and deterministic optimisations are performed on the surrogate model. Different probabilistic optimisation methods, i.e. robust-design optimisation and reliabilitybased design optimisation, are utilised.

The results of the initial deterministic optimisation showed that optimised designs for maximum LBL are achieved by minimising the height and angle and maximising the flange of the stringer. The optimised designs for maximum NPS, on the other hand, are associated with maximised height and angle as well as maximised flange size.

Robust-design optimisations were conducted by considering uncertainties in mechanical properties and geometric 
Fig. 16 Probability density function plot of NPS distribution for the reliable and deterministic stringer designs of masses a $368.5 \mathrm{~g}$ and $\mathbf{b} 391.5 \mathrm{~g}$. The solid and dashed vertical lines show the NPS with a reliability of $\beta_{t}=5.0$ for reliable and deterministic designs, respectively (a)

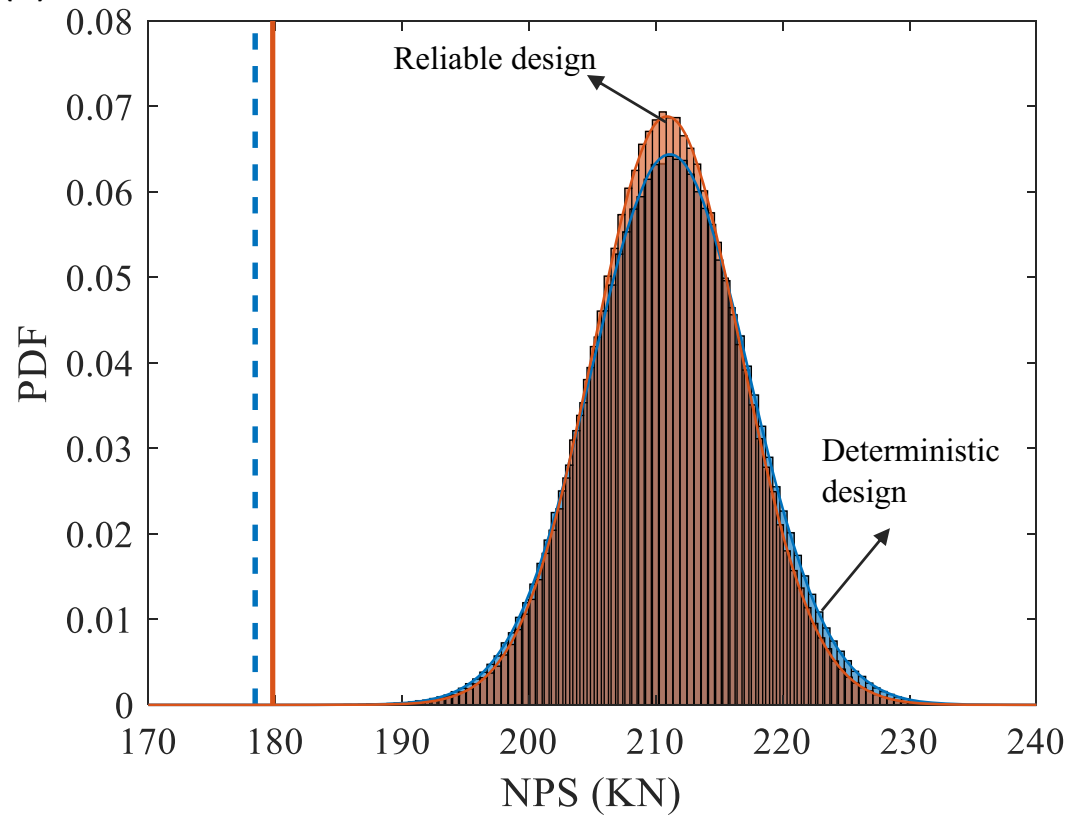

(b)

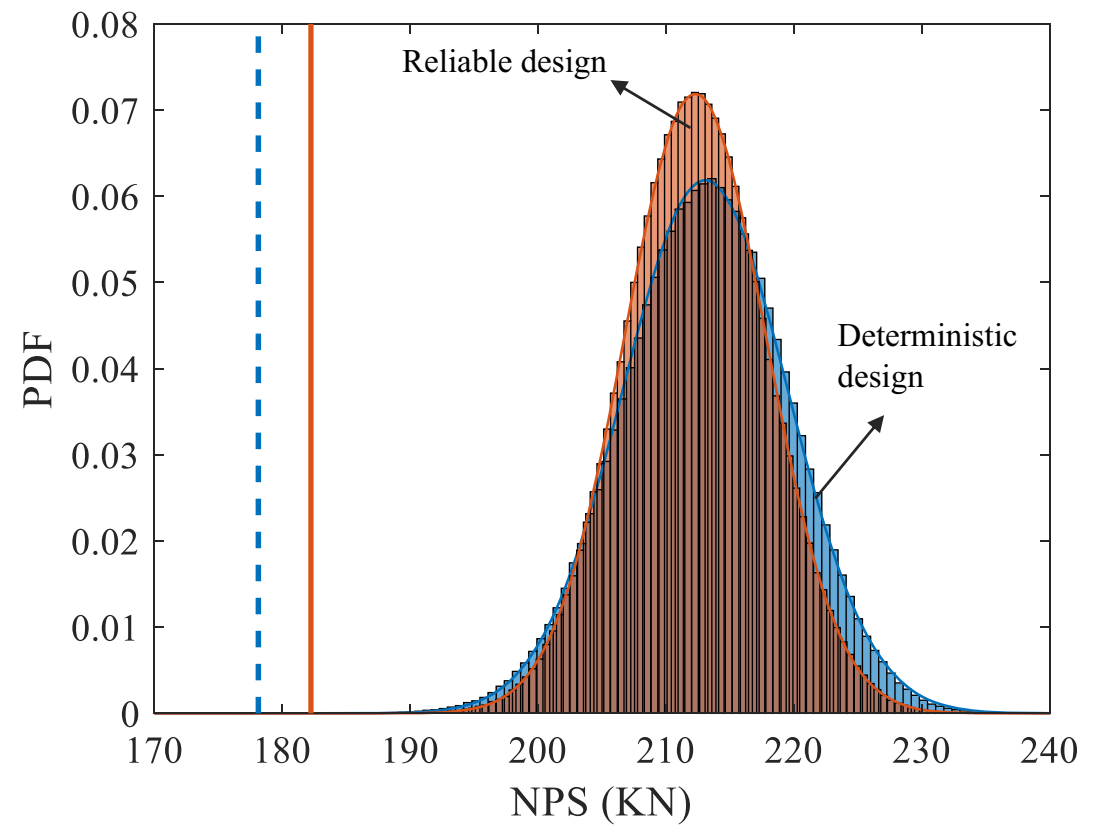

parameters. It was shown that for both cases, compared to deterministic designs, robust designs tend to have slightly smaller NPS mean value and smaller NPS standard deviation. In other words, the RDO finds designs which are less sensitive to variations in mechanical properties and geometric parameters but at the cost of slightly reduced NPS mean value compared to the deterministic designs. Furthermore, RDO designs vary significantly depending on the weights given to the mean and standard deviation objectives.
Finally, reliability-based design optimisation results showed that more reliable designs can be achieved by slightly modifying the stringer angle and height. More specifically, it was shown that the reliable designs offer larger NPS (in the order of several KNs) with a reliability of $\beta_{t}$ while not reducing the NPS mean value much (1 KN or less).

\section{Compliance with ethical standards}

Conflict of interest The authors declare that they have no conflict of interest. 
Replication of results There are several ways through which the readers can check their simulation results against those presented in this study. First is using the tables provided in this study; in fact, the most important optimisation results of the present study are reported using tables including the optimisation inputs and outputs to make it easier for benchmark analysis and comparison purposes. Additionally, the raw data for constructing the results presented in Figs. 4, 7, 9, 11, 13, 14 and 15 is provided as supplementary materials; this allows the readers to check their optimisation results against a wide range of results reported in this study. The size of raw data for Fig. 16 is very large due to the large number of points $\left(10^{6}\right)$ used to create the distribution; therefore, the data for that figure is not provided. Additionally, since the random normal distributions and truncated ones for Monte Carlo samplings (i.e. Figs. 6 and 10) are different each time they are generated, the data for these figures is not provided.

It should be noted that the readers might obtain slightly different results than those presented in this study due to the probabilistic nature of the optimisation as well as the differences in the algorithms used for surrogate model development. If a surrogate model is developed by a reader with a similar accuracy to the one used in this study, even if a different method is used, they should be able to obtain results close to those presented in this study. As explained in the manuscript, this study utilises a Monte Carlo sampling for robust optimisation analysis using $10^{5}$ samples. Such large number of samples was intentionally selected (through a convergence analysis), to ensure a converged probabilistic optimisation analysis assuming random normal distributions.

Open Access This article is licensed under a Creative Commons Attribution 4.0 International License, which permits use, sharing, adaptation, distribution and reproduction in any medium or format, as long as you give appropriate credit to the original author(s) and the source, provide a link to the Creative Commons licence, and indicate if changes were made. The images or other third party material in this article are included in the article's Creative Commons licence, unless indicated otherwise in a credit line to the material. If material is not included in the article's Creative Commons licence and your intended use is not permitted by statutory regulation or exceeds the permitted use, you will need to obtain permission directly from the copyright holder. To view a copy of this licence, visit http://creativecommons.org/licenses/by/4.0/.

\section{References}

Akula VM (2014) Multiscale reliability analysis of a composite stiffened panel. Compos Struct 116:432-440

Albanesi A, Roman N, Bre F, Fachinotti V (2018) A metamodel-based optimization approach to reduce the weight of composite laminated wind turbine blades. Compos Struct 194:345-356. https://doi.org/ 10.1016/j.compstruct.2018.04.015

Bacarreza O, Aliabadi MH, Apicella A (2015) Robust design and optimization of composite stiffened panels in post-buckling. Struct Multidiscip Optim 51:409-422. https://doi.org/10.1007/s00158014-1136-5

Barkanov E, Ozolinšs O, Eglīitis E, Almeida F, Bowering M, Watson G (2014) Optimal design of composite lateral wing upper covers. Part I: linear buckling analysis. Aerosp Sci Technol 38:1-8

Barkanov E, Eglītis E, Almeida F, Bowering M, Watson G (2016) Optimal design of composite lateral wing upper covers. Part II: nonlinear buckling analysis. Aerosp Sci Technol 51:87-95

Bisagni C, Lanzi L (2002) Post-buckling optimisation of composite stiffened panels using neural networks. Compos Struct 58:237-247

Bushnell D (1987) PANDA2 - program for minimum weight design of stiffened, composite, locally buckled panels. Comput Struct 25:469605
Butler R, Williams FW (1993) Optimum buckling design of compression panels using VICONOPT. Struct Opt 6:160-165

Chen X, Qiu Z (2018) Reliability assessment of fiber-reinforced composite laminates with correlated elastic mechanical parameters. Compos Struct 203:396-403. https://doi.org/10.1016/j.compstruct.2018.05.032

Cheung Y (1968) The finite strip method in the analysis of elastic plates with two opposite simply supported ends. Proc Inst Civ Eng 40:1-7

Coello CA (2000) An updated survey of GA-based multiobjective optimization techniques. ACM Comput Surv (CSUR) 32:109-143

Corne DW, Knowles JD, Oates MJ (2000) The Pareto envelope-based selection algorithm for multiobjective optimization. In: International conference on parallel problem solving from nature. Springer, pp $839-848$

Corne DW, Jerram NR, Knowles JD, Oates MJ (2001) PESA-II: region-based selection in evolutionary multiobjective optimization. In: Proceedings of the 3rd Annual Conference on Genetic and Evolutionary Computation. Morgan Kaufmann Publishers Inc., pp 283-290

das Neves Carneiro G, Antonio CC (2018) A RBRDO approach based on structural robustness and imposed reliability level. Struct Multidiscip Optim 57:2411-2429

Díaz J, Montoya MC, Hernández S (2016) Efficient methodologies for reliability-based design optimization of composite panels. Adv Eng Softw 93:9-21

Enevoldsen I, Sørensen JD (1994) Reliability-based optimization in structural engineering. Struct Saf 15:169-196

Fang H, Gong C, Li C, Li X, Su H, Gu L (2018) A surrogate model based nested optimization framework for inverse problem considering interval uncertainty. Struct Multidiscip Optim 58:869-883

Ghiasi H, Pasini D, Lessard L (2009) Optimum stacking sequence design of composite materials. Part I: constant stiffness design. Compos Struct 90:1-11. https://doi.org/10.1016/j.compstruct.2009.01.006

Ghiasi H, Fayazbakhsh K, Pasini D, Lessard L (2010) Optimum stacking sequence design of composite materials. Part II: variable stiffness design. Compos Struct 93:1-13. https://doi.org/10.1016/j. compstruct.2010.06.001

Gorissen D, Couckuyt I, Demeester P, Dhaene T, Crombecq K (2010) A surrogate modeling and adaptive sampling toolbox for computer based design. J Mach Learn Res 11:2051-2055

Hao P, Liu C, Yuan X, Wang B, Li G, Zhu T, Niu F (2017) Buckling optimization of variable-stiffness composite panels based on flow field function. Compos Struct 181:240-255. https://doi.org/10. 1016/j.compstruct.2017.08.081

Hashin Z (1980) Failure criteria for unidirectional fiber composites. J Appl Mech 47:329-334

Hashin Z, Rotem A (1973) A fatigue failure criterion for fiber reinforced materials. J Compos Mater 7:448-464

Herencia JE, Haftka RT, Weaver PM, Friswell MI (2008a) Lay-up optimization of composite stiffened panels using linear approximations in lamination space. AIAA J 46:2387-2391

Herencia JE, Weaver PM, Friswell MI (2008b) Optimization of anisotropic composite panels with T-shaped stiffeners including transverse shear effects and out-of-plane loading. Struct Multidiscip Optim 37:165-184. https://doi.org/10.1007/s00158-008-0227-6

Hillerborg A, Modéer M, Petersson PE (1976) Analysis of crack formation and crack growth in concrete by means of fracture mechanics and finite elements. Cem Concr Res 6:773-781. https://doi.org/10. 1016/0008-8846(76)90007-7

$\mathrm{Hu}$ N, Duan B (2018) An efficient robust optimization method with random and interval uncertainties. Struct Multidiscip Optim 58: 229-243

Irisarri F-X, Laurin F, Leroy F-H, Maire J-F (2011) Computational strategy for multiobjective optimization of composite stiffened panels. Compos Struct 93:1158-1167

Kassapoglou C (2013) Design and analysis of composite structures: with applications to aerospace structures. Wiley. https://doi.org/10.1002/ 9781118536933 
Kaveh A, Dadras A, Malek NG (2018) Robust design optimization of laminated plates under uncertain bounded buckling loads. Struct Multidiscip Optim 59:877-891

Koziel S, Ciaurri DE, Leifsson L (2011) Surrogate-based methods. In: Computational optimization, methods and algorithms. Springer, pp 33-59. https://doi.org/10.1007/978-3-642-20859-1_3

Lamberti L, Venkataraman S, Haftka RT, Johnson TF (2003) Preliminary design optimization of stiffened panels using approximate analysis models. Int J Numer Methods Eng 57:1351-1380

Lanzi L, Giavotto V (2006) Post-buckling optimization of composite stiffened panels: computations and experiments. Compos Struct 73:208-220

Lapczyk I, Hurtado JA (2007) Progressive damage modeling in fiberreinforced materials. Compos A: Appl Sci Manuf 38:2333-2341

Liu B, Haftka RT, Akgün MA (2000) Two-level composite wing structural optimization using response surfaces. Struct Multidiscip Optim 20:87-96

López C, Bacarreza O, Baldomir A, Hernández S, Ferri H, Aliabadi M (2017) Reliability-based design optimization of composite stiffened panels in post-buckling regime. Struct Multidiscip Optim 55:11211141. https://doi.org/10.1007/s00158-016-1568-1

Marhadi K, Venkataraman S (2008) Surrogate measures to optimize structures for robust and predictable progressive failure. Struct Multidiscip Optim 39:245. https://doi.org/10.1007/s00158-0080326-4

Marín L, Trias D, Badalló P, Rus G, Mayugo J (2012) Optimization of composite stiffened panels under mechanical and hygrothermal loads using neural networks and genetic algorithms. Compos Struct 94:3321-3326

Marler RT, Arora JS (2004) Survey of multi-objective optimization methods for engineering. Struct Multidiscip Optim 26:369-395

Montoya MC, Costas M, Díaz J, Romera L, Hernández S (2015) A multiobjective reliability-based optimization of the crashworthiness of a metallic-GFRP impact absorber using hybrid approximations. Struct Multidiscip Optim 52:827-843

Nikbakt S, Kamarian S, Shakeri M (2018) A review on optimization of composite structures. Part I: laminated composites. Compos Struct 195:158-185. https://doi.org/10.1016/j.compstruct.2018.03.063

Park J-S (1994) Optimal Latin-hypercube designs for computer experiments. J Stat Plann Inference 39:95-111

Qu X, Haftka RT (2003) Reliability-based design optimization of stiffened panels. In: Uncertainty Modeling and Analysis. ISUMA 2003. Fourth International Symposium on, 2003. IEEE, pp 292-297

Rikards R, Abramovich H, Kalnins K, Auzins J (2006) Surrogate modeling in design optimization of stiffened composite shells. Compos Struct 73:244-251. https://doi.org/10.1016/j.compstruct.2005.11. 046

Salas P, Venkataraman S (2008) Laminate optimization incorporating analysis and model parameter uncertainties for predictable failure. Struct Multidiscip Optim 37:541. https://doi.org/10.1007/s00158008-0259-y

Sobieszczanski-Sobieski J, James BB, Riley MF (1987) Structural sizing by generalized, multilevel optimization. AIAA J 25:139-145
Sohouli A, Yildiz M, Suleman A (2018) Efficient strategies for reliabilitybased design optimization of variable stiffness composite structures. Struct Multidiscip Optim 57:689-704

Strömberg N (2017) Reliability-based design optimization using SORM and SQP. Struct Multidiscip Optim 56:631-645

Turon A, Dávila CG, Camanho PP, Costa J (2007) An engineering solution for mesh size effects in the simulation of delamination using cohesive zone models. Eng Fract Mech 74:1665-1682. https://doi. org/10.1016/j.engfracmech.2006.08.025

Venkataraman S, Haftka RT (1999) Optimization of composite panels-a review. In: Proceedings-American Society For Composites. pp 479 488

Venkataraman S, Salas PA (2007) Optimization of composite laminates for robust and predictable progressive failure response. AIAA J 45: 1113-1125. https://doi.org/10.2514/1.22077

Wang D, Abdalla MM (2015) Global and local buckling analysis of gridstiffened composite panels. Compos Struct 119:767-776

Wang D, Abdalla M (2016) Buckling analysis of grid-stiffened composite shells. In: Design and analysis of reinforced fiber composites. Springer, pp 1-18. https://doi.org/10.1007/978-3-319-20007-1_1

Wang D, Abdalla MM, Zhang W (2017) Buckling optimization design of curved stiffeners for grid-stiffened composite structures. Compos Struct 159:656-666. https://doi.org/10.1016/j.compstruct.2016.10. 013

Wang D, Abdalla MM, Zhang W (2018) Sensitivity analysis for optimization design of non-uniform curved grid-stiffened composite (NCGC) structures. Compos Struct 193:224-236. https://doi.org/ 10.1016/j.compstruct.2018.03.077

Wang D, Abdalla MM, Wang Z-P, Su Z (2019) Streamline stiffener path optimization (SSPO) for embedded stiffener layout design of nonuniform curved grid-stiffened composite (NCGC) structures. Comput Methods Appl Mech Eng 344:1021-1050. https://doi.org/ 10.1016/j.cma.2018.09.013

Wind JW, Perdahcioğlu DA, de Boer A (2008) Distributed multilevel optimization for complex structures. Struct Multidiscip Optim 36: $71-81$

Wittrick W (1968) General sinusoidal stiffness matrices for buckling and vibration analyses of thin flat-walled structures. Int J Mech Sci 10: 949-966

Yang N, Das P, Blake J, Sobey A, Shenoi R (2013) The application of reliability methods in the design of tophat stiffened composite panels under in-plane loading. Mar Struct 32:68-83

Youn BD, Choi KK, Park YH (2003) Hybrid analysis method for reliability-based design optimization. J Mech Des 125:221-232

Zabinsky ZB (1998) Stochastic methods for practical global optimization. J Glob Optim 13:433-444

Zitzler E, Deb K, Thiele L (2000) Comparison of multiobjective evolutionary algorithms: empirical results. Evol Comput 8:173-195

Publisher's note Springer Nature remains neutral with regard to jurisdictional claims in published maps and institutional affiliations. 